QK329

.S75

Strobi, Pater Gabriel

Aus der Frühlings-Flora und Fauna Illyriens 


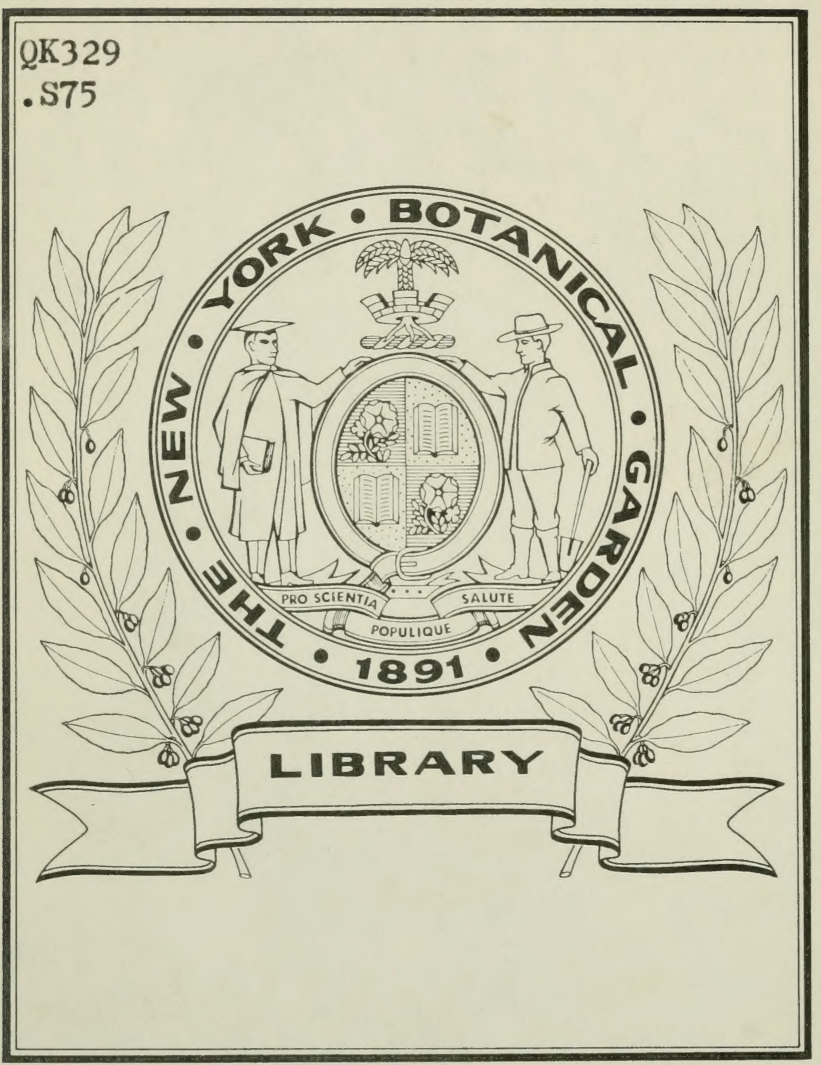




Itrobl 9. . , Aus der Fruilings, lora und Fauna Fllyrions : Terhandlungen d. R.k. zoo bogisch = botanischen Gesell, schaft, Tharg. is F 2 , XXIIsd., Nien is $72 p .577-6 i 6$. 



\section{Aus der Frühlings-Flora und Fauna Illyriens.}

Von

Pater Gabriel strobl,

Benediktiner in Admont.

(Vorgelegt in der Sitzung vom 3. Juli 1872.)

R

Ritter v. Josch, jub. Landesgerichtspräsident und langjähriger Botaniker, hatte im Frühlinge des Jahres 1871 mit mir eine Reise vach Illyrien unternommen und Rittẹr v. Tommasini, der allbekannte "Vater der istrianischen Flora", hatte in St. Peter sich uns angeschlossen und während eines grossen Theiles derselben uns begleitet. Er war, wenn auch nicht der Urheber, so doch lange Zeit der Leiter unseres Unternehmens, indem er die Punkte auswählte, die wir als die lohnendsten mitsammen besuchten oder nach seiner Trennung noch besuchen sollten, und, was uns noch mehr als dieses förderte, er bestimmte uns auch Tritt für Tritt die gesammelten Pflanzen. Es ist daher nicht mehr als billig, wenn ich an dieser Stelle ihm nochmals unseren innigen Dank für seine aufopfernde Freundschaft ausspreche und einen grossen Theil des Verdienstes, welches ich mir etwa durch diese Arbeit erwerbe, ihm zuschreibe. Doch darf ich nicht verschweigen, dass auch Herr v. Josch in diesem Florengebiete sehr bewandert war, da er einen Theil desselben wiederholt besucht und sogar in der österr. botan. Zeitschrift 1863 einen längeren Aufsatz darüber veröffentlicht hatte. Endlich habe ich selber alles Gesammelte zu Hause sorgfältig durchgearbeitet und mit Hilfe botanischer Werke, besonders der ausgezeichneten Abbildungen in Reichenbach's "Flora Deutschlands" nochmals determinirt. Meine versuchsweisen Bestimmungen der Moose wurden durch die Güte des Herrn J. Juratzka berichtigt und die bei den Käfern zweifelhaften Arten übernahm mit gleicher Freundlichkeit Herr L. Miller, beide bekanntlich in Wien. Herr v. Tommasini hatte auch die Güte, die nach Vollendung des Aufsatzes noch stehen gebliebenen 


\section{.575}

Dubia nach bestem Vermögen aufzulösen. Zur Hebung etwa noch auftauchender Zweifel behielt ich die gesammelten Pflanzen und Käfer vollzählig beisammen und bilden dieselben einen Theil der naturhistorischen Sammlungen zu Admont, welche allerdings, da die alten durch den schrecklichen Brand anno 186 s vernichtet wurden, noch ziemlich in den Windeln liegen. Viel bessere Aufschlüsse, als das meine, bietet übrigens das Riesenherbar v. Tommasini's zu Triest und die stete Anwesenheit seines diensteifrigen Besitzers.

Unsere Reise durch Illyrien dauerte vom 29. April bis 18. Mai und umfasste einen Theil Krains, des kroatischen Küstenlandes nebst dem Scoglio San Marco, die quarnerischen Inseln Veglia, Cherso, Lossino oriule und Pietro di Nembi, das istrianische Küstenland von Pola bis Promontore und die Umgebung der Stadt Triest. Die gesammelten Gegenstände waren vorzugsweise Phanerogamen und Käfer, bei welch' letzteren mich die Umsicht des in entomologischen Kreisen wohlbekannten Dr. Kriechbaumer aus München, der von Fiume bis Cherso uns begleitet hatte, bedeutend unterstützte. Der mir von hochachtbarer Seite zugekommenen Aufforderung, ein „allgemein naturwissenschaftliches Bild“ zu liefern, entsprechend, werde ich mich nicht auf eine trockene Aufzählung des Gesehenen und Gesammelten beschränken, sondern den allgemeinen Charakter des Landes nit dem der Flora, so gut ich es vermag, verbinden, mich aber streng auf das Gebiet des Zoologisch-Botanischen beschränken. Wer sich auch für die auf unserer Reise geschauten Panoramen, Culturzustände, Städte, Schiffe, Bewohner etc. interessirt, mag: sich aus meiner "Frühlingsreise nach Süden" (Graz 1872, Verlag der Vereinsdruckerei, $256 \mathrm{~S}$., $80 \mathrm{kr}$.) näher informiren.

\section{Allgemeine Jebersicht. ${ }^{1}$ )}

Im Süden der Centralkette erhebt sich die schroffe, bis $6000^{\circ}$ hohe Kette der Karawanken, an die sich südöstlich die Kalkalpengruppe der Steirer oder Sulzbacher Alpen, südwestlich die des Terglou-Stockes anlehnt. Von Letzterem zieht sich (unter verschiedenen Namen) eine gewaltige Reihe theils plateau-, theils kettenförmiger Züge nach Südosten und erfüllt den grössten Theil von Krain, Istrien, Kroatien und Dalmatien; selbst die Inseln des Quarnero und Dalmatiens sind nichts anderes als die letzten, ebenfalls südöstlich streichenden und über die

1) Besonders nach Schaubach: "Die deutschen Alpen" V. B. 1, Stur: "Ueber den Einfluss des Bodens" auf die "Vertheilung der Pflanzen", Sitzungsber. d. k. k. Akad. d. Wissensch. 1856 u. 57, Neilrei ch: „Die Vegetationsverhältnisse von Kroatien ", herausgegeben von der k. k. zool.-bot. Gesellsch. 1868 und "Flora von Nieder-Oesterreich“, Cotta: "Die Alpen“ 2. Ausgabe, 1851. 
Fluthen erhobenen Vorlagen dieses Felsgebirges, mit dem sie unterseeisch zusammenhängen. Der dem Terglou zunächst liegende Theil dieser Höhen führt den Namen ,julische Alpen“ und bildet einen breiten Gürtel zwischen dem Thale der Save, dem Meere und dem Isonzo; doch führt er seinen Namen sehr mit Unrecht, denn das von ihm gebildete Plateau erhebt sich höchstens 2000 über das Meer und kaum 1000` über das Laibacherfeld. Der südliche Theil dieses Plateaus oder der Karst (Carso) im engeren Sinne hat etwa eine Höhe von $14-1500^{\prime}$ und breitet sich in ermüdender Einförmigkeit zwischen Triest und St. Peter aus; südöstlich vom Karste läuft das Gebirge terrassenförmig aufsteigend vom Westen nach Osten bis $3000^{\prime}$ und darüber und bildet die sogenannte Tschitscherei (čičerie), mit welchem Namen Manche auch das ganze Hochplateau von Istrien bezeichnen; der südöstliche Hochpunkt derselben ist der ganz Istrien überschauende Monte Maggiore (4410); östlich fällt das Gebirge steil ab, gegen Süden aber senkt es sich allmälig zu den beiden Seiten des istrianischen Dreiecks nieder, als dessen Grundlinie man die Tschitschenkette betrachten kann, und verläuft besonders gegen Westen ziemlich flach. Auch auf den Hauptinseln des Quarnero finden sich mehr oder minder hohe Spitzen und Plateaux; so besitzt Veglia den Monte Triskovaz $\left(3678^{\circ}\right)$, Cherso den M. Syss (2016') und das über 1000' erhobene Plateau der "Arabia petraea“, Ossero den M. Ossero (1842') und selbst das kleine Pietro di Nembi den Monte Grisina (circa $600^{\prime}$ ). Noch viel bedeutender sind die uns nicht näher berührenden Hochgipfel des kroatischen Vellebith und die dalmatinischen Felsenkämme.

Diese Hochplateaux und Felsenketten aber bilden kein ununterbrochenes Ganze, sondern werden mebrfach von Mulden und weiten Becken durchschnitten. So schiebt sich im Norden zwischen die Karawanken und die julischen Alpen die grosse, vielgegliederte Ebene von Oberkrain, welche zur Mittel-Tertiärzeit eine tiefe Bucht des ungarischen Meeres war; eine 19 Meile lange Mulde verläuft von Görz durch das Wippachthal und, den Tschitscherboden von der Hochebene des Schneeberges (5673') scheidend, durch das tiefe Reccathal bis zum Dvetroberge, um jenseits desselben als schmaler Kanal durch das Reczina- und Dragathal über Buccari nach Novi zu streichen, stets in südöstlicher Richtung, und eine zweite, noch breitere, in der Mitte aber von einem Karstzweige diagonal durchschnittene Mulde - die Istrianer Mulde - geht durch das eigentliche Istrien vom Fusse des Karstes, ebenfalls in südöstlicher Richtung, bis an die Südostküste bei Albona.

Was nun die Gesteinsart betrifft, so bestehen die Hochplateaux und Felsenkämme durchwegs aus einem hellen, dichten Kalksteine, und zwar herrscht nördlich von Oberlaibach die obere Trias-, südlich davon die Kreideformation. So besteht der Birnbaumerwald, das Waldgebirge des Schneeberges, der eigentliche Karst, der nordwestliche Tschitschenboden, 
der südőstliche mit dem M. Maggiore, der westliche Istrianerkarst, das kroatische Küstenland u. s. w. ganz aus Hippuritenkalk, an den Rändern desselben und der Mulden aber findet sich der dem jüngeren Eocän angehörige Nummulitenkalk, z. B. bei Opschina am Recca- und ReczinaThale. Alle diese Kalkgesteine sind untereinander und von den Alpenkalken, als deren südlicher $Z$ weig ja das Ganze gelten muss, in petrographischer Hinsicht nicht bedeutend verschieden und zeigen daher diese Ketten so ziemlich dieselben Erscheinungen wie die Kalkalpenketten der Karawanken, des Dachsteinzuges, der Salzburger, Tiroler und baierischen Alpen, nur dass hier die allen gemeinsamen Eigenschaften noch greller und auffallender hervortreten. Wie am "steinernen Meer" des Dachsteinzuges oder am „todten Gebirg“" bei Aussee, so sehen wir auch hier auf den welligen Plateaux unübersehbare, nackte Steinfelder, eckige Felstrümmer, scharfgeschnittene Kämme, schroffe Abstürze, spitz aufragende Kegel, mit Geröll oder Schutt überdeckte Abhänge, tiefe Spalten, kesselförmige "Erdfälle" oder trichterförmige Vertiefungen. Vor Allem aber gleichen sich all' diese Gebirge in ihrem Höhlenreichthum und ihreu (unentwickelten) Flusssystemen. Alle diese Kalksteine sind der Einwirkung: des Wassers sehr zugänglich, weniger aber in der Weise, wie die Schiefersteine, welche verwittern und fruchtbare Krume bilden, sondern vielmehr nur insofern, als sie, vom Wasser immer mehr zerklüftet und zerbröckelt, in einzelne, grössere oder kleinere Stücke zerfallen, welche dann öde Steinfelder bilden oder, besonders in den Hochgebirgen, als Schutthalden die Felsen umlagern. In diesen Klüften versinkt das atmosphärische Wasser nach kurzem Laufe und sucht sich unterirdisch einen Ausweg, das Plateau selber aber bleibt auf diese Weise oft meilenweit dürr und trocken. So gibt es Gewässer, die plötzlich als starke Quellen hervorbrechen, eine Zeit lang durch eine Mulde fortlaufen, dann wieder ebenso plötzlich versinken, um anderswo wieder aufzutauchen; ein solcher ist die Laibach, welche bei Sagurie als Poik entspringt, bei Adelsberg sich in die berühmte Grotte wirft, bei Planina als Unz aus der prachtrollen Unzgrotte hervorströmt, eine Meile lang in Serpentinen sich herumwindet, und dann wieder in einer Grotte sich birgt, um endlich nach dreistündigem Laufe bei Oberlaibach als Laibach herauszutreten und ihre Fluthen in die Save zu führen. - Andere Gewässer treten gleich anfangss als schiffbare Flüsse aus den tiefsten Stellen der Berge hervor, wie die träge Ischza und Bistra am Rande des Laibacher Moores, wieder andere sind blosse Küstenflüsse, die nach äusserst kurzem Laufe sich in das Meer ergiessen, wie die Reczina bei Fiume oder der gewaltige Timavo bei Duino, auf welchem die Seeschiffe sogar bis zur Quelle fahren; ähnlich brechen am Fusse des M. Maggiore zahlreiche Quellen hervor, von denen das trockene Istrien bewässert wird und kaum minder reichlich sprudeln die Süsswasserquellen am Gestade bei Aurosina, Fiume, und besonders zwischen 
Buceri und Bucerizza. - Arhnlich, wie mit den Flïssen, rerhait es sich auch bei den Seen; es gibe deren nur wenige oberirdische und die es gibt, sehen wir ohne sichtharen Zu- und Abiuss, so den Zirknither See in Krain, den Cepich-See in Istrien, den Lago di Vrana auf Cherso.

Mit dem öden, trockenen Charakter des Gesteims hängt zusammen das Auftreten der liora, besoulers seithem dureh die selhataiehtige Unvorsichtigkeit der a!tron Venetianer die Möhen ron dem schïzenden Nadeiund Laubwerke grosentheils entblümt wurlen. Die furchtbare fiewalt dieses oft orkanartigen, kalten und trockeneen Fordostwindes ist nur allzu bekannt und ehenso anch scine verdepbliele Wirkung, dass er nämlich die lockere Dammerde, wehehe aut kalk ohmehin nur spärlich - in Felsspalten oder tieferen Grïnden — sich ansiedele, grossentheils fortrafle und daher das Anlegen von Fruehteddern anil den Plateaux ganz unmöglich macht. In Cherso sind selbst starke Steineichen und Wachholderbäume ob seines Anpralls ganz wagrecht gebogen, ja sogar fast gänzlich geschlusene dleeresbuchten, wie die ron isucari, iuhlen sein Wüthen und werfen dann hausiolie Wellen. Beror das Land jene grossartigen Entblïsungen getroffen hatten, war das Klima noch etwas feuchter, da bekanntlich die liälder stets die Feuchtigkeit länger festhalten, jetzt aler ist das Klima troken, ausserordentlich trocken, besonders auf den lnseln, so dass daselbst ofters melrere Monate laig kein Regen fällt und da ist nun die Bora der traurige Vermittler und Ausgleicher les grellen Wechsels zwischen plötzlicher Fenchigkeit und uncrträglich I Itze. Mögen die Bembihungen der wakkeren Triestin r, wehe die Karsthöhen rings um die Stadt mit Sehwartöhren-Wäldern besetzen, bald auch auf die übrigen Plateaux sich erstrecken und ihnen so das alte Klima wiederbringen!

Dass unter den obwaltenden Umständen die Flora nicht allzu reichlich sein kann, lässt sich leicht ermessen. Int die Vegetation des Kalkfelsens, gleichviel, wessen Alters er sei, gegenüher dem erfreulichen Grün und den üppigen Matten der Schieferberge, wenn auch reicher an Arten und Seltenheiten, dennoch an fndividuenzahl unendlich ärmer, da eben der Kalk schwer verwittert und die Krume mcist nur in Felsspalten und tieferen Gründen sich sammelt, so muss sie bei der noch grüsseren Zerrissenheit des Kreidekalkes und den steten Angriffen der Bora noch viel kümmerlicher ausfallen und die I'llanzen kïnnen sich ausschlsesslich nur aus den Xerophilen recrutiren. Yon einer ununterhochenen Wiesendecke kamn nirgends eine Rede sein, denn jede Gelegenheit zur Bildung eines nach stur dazu unumgänglich nothwendigen, aus Kalk-, Kiesel- und Thon-Erde gleichmïsig gemischten Bodens fehlt wegen Mangel an Silicatgesteinen gänzlich und auch ron Laub-oder Nadelwaldern finden sich nur selten grö̈ssere, dichte Complexe. Nur kïmmerliches Gesträuch oder rerkrüpelnde Waldbáume rermögen sich auf den düren, wascerlosen

14. Xill. dbhand, 
Höhen zu halten und dazwischen spärliches Graswerk, kaum hiureichend für die genügsamen Schafe. Ja manchen Gegenden fehlt auch dieses, und sie wïrden ganz öde daliegen, wenn nicht die Vorsehung auch dort etwas geschaffen hätte, wohin sich der Mensch mit seinen Werken flüchten kaun, die Dolinen. Es sind diess mehr oder minder grosse, kesselförmige Vertiefungen, denen die Bora das Erdreich nicht zu rauben vermochte und in welchen sich daher das zusammensickernde Wasser länger halten kamn. Doch sind auch diese meistens noch mit einer schützenden Mauer umfangen, zu welcher man die Steine vom Boden der Doline aufgelesen, und erst der innere Raum, die sogenanute Ograda, zeigt prangende Saaten oder Weinreben. In der Nähe dieser Dolinen, wenn nicht in ihnen selber, sieht man auch oft die Wohnhäuschen, aus grauen Kalkstein aufgeführt und häufig auch uit grauen Kalksteinen gedeckt, als wären sie ebenfalls Karstgebilde. In der vollen, so eben geschilderten Traurigkeit treten uns zum Glïcke nur der Karst im eigentlichen Sinne zwischen Sessana und Nabresina, die Tschitscherei und die "Arabia petraea" entgegen. Der sogenaunte "Waldkarst" von Laibach bis St. Peter, sowie überhaupt der krainerische Antheil des Karstes ist vielfach bewaldet und seine Weiden sind viel freundlicher, die südlichen, geschützten Abfälle der Gebirge aber, besonders bei Fiume und Triest, sowie ein grosser Theil von Veglia und Ossero sind oft ausserordentlich schön belaubt und durch die Hand des Menschen fast zu einem Paradiese umgeschaffen. Doch ist im ganzen Kalkgebiete, besonders auf den Inseln, der Getreidebau selten, denn das Getreide foruert gleich den Wiesenmatten einen aus den drei Erden gleichmässig gemischten Boden, welchen die Kalkkrume nicht zu bieten vermag; dafür aber gedeiht hier desto üppiger der xerophile Weinstock, die Olive, der Feigenbaum, immergrïne Eichen und andere Südgewächse.

Anders verhält es sich in den oben erwähnten Mulden und dem grossen Tertiärbecken, denn dort ist auch das Gestein ganz ein anderes. Die grosse Ebene ist überdeckt von der gleichmässig gemischten Dammerde der Meeresablagerungen, nur der südlich von Laibach liegende Theil - das Laibacher Moor - wird erfüllt mit recenten Torfbildungen; aus dem Becken aber erhebensich grüne Triasschiefer-Berge, z. B. der Schlossund der Grosskahlenberg. - Die Mulden und ihre Verbindungskanäle gehören zum Eocän und bestehen aus Mergeln und Sandsteinen; auch bei Triest ist, besonders gegen den M. Spaccato hinauf und längs der Eisenbahnlinie gegen Westen, sehr viel Sandstein autgeschlossen. Hier entwickelt sich eine ganz andere Flora; denn der leicht verwitternde Sandstein und Schiefer gibt seines thonig-kiesigen Gehaltes wegen eine reiche, fruchtbare Dammerde und besitzt eine beträchtliche Feuchtigkeit, wesshalb er sich mit hygrophilen Pflanzen bevölkert und sich über ihm eine freudig grümende, üppige Decke bildet oder hohe schattige Laubbäume 
ihre Kronen erheben. So ist diese Flora reich an Indiriduen, aber arm an eigenthümlichen Arten und Seltenheiten. Die Dammerde ist, da die Mulden und das Becken von den Kalkhöhen mit Kalkerde reich versorgt wurden, mit allen drei Erden hinreichend versehen und so sind diese Mulden gleich dem grossen becken auch der IIauptsitz des Getreidebaues; selbst das Moor wird allmälig durch das fortwährende Ausbrennon und Kanalisiren entsumpft und in eine fruchttragende Flache verwandelt.

Nach Bartling's Dissertatio de littoribus et insulis maris liburnici theilt sich die Flora der von uns besuchten Jänder in drei Recrionen, die der Myrthe, der Manma-Esche und der Buche-Fichte, welch' letztere er die alpestre nennt. Die biden ersten Regionen gehören der Mittelmeerflora an, die dritte aber umfasst die inneren Hochplateaux und die Fiebirge. In der ersten sind die immergrïnen Laubhölzer zu Hause, besonders also die Myrte, welche der Region den Namen gab, verschiedene Eichen, Cistusarten, Pistaceen, Phyllyreen, Lorbeer, Olive, Feige und andere, ron Nadelhölzern besonders die acclimatisirte Pinie, Cypresse, rothbeerige Wachholderbäume, in den Gärten Citronen-, Caroben-, Granatäpfel-, bisweilen sogar Dattelbäume. In diese Region gehören unsere Excursionen auf Cherso, Ossero, Oriule, Pietro di Nembi, ron Pola bis Promontore, theilweise auch schon Veglia. Die zweite Region umfasst meist uur sommergrïne Laubbäume und in ihr findet sich vor Allem dic Manna-Esche, die Flaum- und Zerr-Eiche, die Hopfen- und die Duiner Buche, die echte Kastanie, der Perrïckenstrauch und der äusserst gemeine Paliurus; doch reichen manche der ersten Region, besonders Oel- und Feigenbiiume, anch in diese herein. Zu dieser Region gehört der grösste Theil ron Istrien nördlich von Dignano und das eigentliche Littorale, somit die ron uns besuchten I'mgebungen Fiume's, Triest's, San Marco und Veglia. In diesen beiden Regionen sind besonders vertreten die Formen der Labiaten, Asperifolien, Papilionaceen, die besonders im Sommer charakteristischen der Umbelliferen und Disteln, in der zweiten auch die der Liliaceen. Sie sind ferner der Sitz des Feigen-, Oliven- und Treinbaues, aber es fehlen ihmen, besonders der ersteren, dichte, hochstämmige Wälder und saftig-grüne Wiesen fast gänzlich, die Höhen sind meist mit Buschwerk besetzt und ron somnigen Gras- oder Steinflächen unterbrochen. In pflanzengeographischer Hinsicht gehören sie zur "wärmeren, gemässigten Zone." Die dritte Region endlich trägt ganz mitteleuropäischen Charakter und enthält daher die bei uns gewöhnlichen Waldbäume, z. B. Buchen, Hainbuchen, Tannen, Fichten, an der Grenze natiurlich noch gemischt mit manchen südlichen Formen. In dieser Region haben wir nur sehr wenig gesammelt und zu ihr dürfte aus unserer Reise bloss der Tebergang ïber die gjulischen Alpen" und die Lmgoinung Laibach's gehören. Pflanzengeographisch gehört sie zur ,kälteren, gemässigten Zone" oder zur "küblen Region." 
Betrachten wir noch die von uns gesammelten Pflanzen mach der Verschiedenheit des Standortes, so gehören die meisten in die "Vegetationsform der steinigen oder felsigen Hügel und buschigen Stellen," mit welcher in diesem Gebiete die "der Weiden" so ziemlich zusammenfällt, ferner in die , des bebauten Landes", da die Aecker, Weingärten und Olivenpflanzungen überaus reich an seltenen Pflanzen - natürlich meist Cnkraut - sind, dann in die ,der unfruclutbaren Raine und wïsten Plätze", deren es im Ganzen wohl gar viele gibt, besonders die Auschüttungen bei Fiume und die Umgebung des Städtchens Ossero; die ,der Wälder", wozu theilweise die Umgebung von Fiume, das "Boschetto" auf Veglia und der "Kaiserwald" bei Pola gehört; die "des Seestrandes" mit den eigenthümlichen Salzpflanzen; die ,der Wiesen", die wir in grösserer Ausdehnung nur bei Laibach und am Prà grande bei Pola fanden; endlich die höchst spärliche ,der Sümple", nämlich ebenfalls bloss bei Laibach und stellenweise auf Veglia, Ich unterlasse es, die in jede dieser Formen einschlägigen und olt in andere Formen überspringenden Phanzen zu nennen, da ich sie auf meiner späteren Reiseschilderung wiederholen müsste und gehe nun über zur eigentlichen Arbeit, nämlich zur chronologisch-topographischen Aufzïhlung der auf unserer Reise gesammelten oder wenigstens notirten Pflanzen und Käfer. Ich werde das Ganze in einzelne, möglichst selbstständige und abgerundete Theile zerleg*en und anch stets den Gesammt-Charakter des Terrains in einigen Zügen skizziren. Möge die Arbeit ein tauglicher Beitrag werden zu einer künftig’en Flora Illyriens.

\section{Laibach.}

Nach Krain, dem ersten Ziele unserer botanischen Thätigkeit, drangen wir durch die Thermopylen, welche dieses Land mit der benachbarten Steiermark verbinden. Wer ist vor diesen schauerlichen Felsenzimnen mit ihren kahlen, weissgebleichten Wänden und ihren grotesken, fantastischen Formen, welche ron Steinbrück bis gegen Littay fast ununterbrochen in gleicher Grosirtigkeit sich aufbauen, nicht erschrocken, wenn die Locomotive eilenden Fusses an ihnen vorïberflog und tief unten die Save in unheimlicher Schnelle ihre grïnlichen Fluthen wälzte! Und doch war für uns ilir Anblick froh und herzerhebend; war ja diese Enge das Thor zum Süden und hatte ja auch hier der Lenz seinen Einzug gehalten und selbst den harten Felswïnden manches seiner Blïtenkinder aufgedrungen. So sahen wir in Menge von den Wänden das blaue Elfengras (Sesleria coerulea $\mathrm{Ard}$.) herunternicken oder die gelben Blüthen des Wundklees (Antlyllis Vulneraria L.), des Leontodon incanus Schrk. und der brillenschotigen Biscutella laevigate L., ja der haarig'e Ginster (Genista pilosa r.) Überzog sogar weite Strokm mit seinen goldigen Bliithen; am 
gewimnendsten aber lachten uns entgegen die tausend und aber tausend Purpurblïthen des Iradenröschens (Eviea cornea I..) und die zwar blatterlose, dafiir aber mit zahleeichen, milchweissen Blumen ïber:äete Felsenmispel (A.onia rotmmetioli, l’rs.); hie und da neben den seltenen Iräuschen winkte auch das zarte Lauh des Weinstocks oder die Rosabliithen des P'firsichbaumes. Bei Sara bliei ob der steten Steigung der Weinstock zuriick, aber auch die Fclsen begannen allmailig zu weichen und statt des kleinen Strïplwerks auf kahlen Ï̈hen erhoben sich auf den grïnen, sanft gerundeten IIïgeln Erlen, Eschen und stafliche Buchen, bis auch diese schwanden und wir die write, rejel bebaute, von den hohen, sïdlichen Kalkalpen umstandene Ebene durcheilend nach Labach kamen.

Unser erster Gang galt dem Laibacher Felde an der Westgrenze der Inuptstadt. Wir promenirten durch den Stadtpark, wellher seinen Schatten gleich dem Grazer Glacis prächtigen, wilden Kastanionbäumen verdankt und kamen in der Nähe des freundlichen Schlosses Tiroli in's Freie. Meine Hoflnung auf' reiche Ausbeute wurde aber sowohl hier als auch läng's des nahen Wraldsames arg hetrogen. Mag die im Allgemeinen zuriickqebliebene Entwickelung, oder der tertiäre Bodencharakter, oder die Nähe der Cultur, oder Alles zusammen daran Schuld gewesen sein, genug, wir fanden fast durchgehends nur fiemeines und auch von diesem nicht gar Vieles: 1) "Tiola canina L., * Cutisus hirsutus L., Pencedanum Oreoselinum Mneh., *Galium vernum I.., *Valerianella olitoria Poll., Centuriea axillaris WV., "Symmhtum ofjicinale L., "Lysimachir punctata L., *Euphorbia verrucosa I, an. und *Salia aurita L

Von da gingen wir in den neben der Tirnauer Vorstadt gelegenen Stadtwald, einen kleinen, trotz vielen gegentheiligen Bemuihungen noch inmer sehr sumpfigen und daher für Botaniker viel versprechenden Stieleichenwald. Wir trafen hier auch wirklich mehrere interessante Sumptpflaizen, hesonders die schr häufige, aber meist schon verblühte Fitillariu Melea!ris I., die nicht minder gemeine *Viola uliginosa Sehrad. und das ebenfalls häufige "Leucojum aestium L. Ausserdem eine ziemliche Menge ron Riedgrïsern (*area Davalliana Sm., *stricta Good., T!llanca Scop., *distans L.), einige Weiden (S. "† amygilalina L. F. concolor, teinerea L.), "Ramunculus auriromus L., *Orchis Morio L., Hymum cuspielatum L. und aduncem L.

Von da durch die Tirnauer Vorstadt zur Cesta na grad und auf den Schlossberg. Im Sommer mag dieser Berg des Botanikers Mühe reichlich lohnen, denn die Kunst hat sich an iln noch wenig rersucht

1) Die mit Sternchen rersehenen Phancrogamen waren in Blïthe. die mit $\leftarrow$ bezeichneten durelıwegs oler doch theilweis, in Samen, $\leftarrow=$ blühende nebst schon in Samen stehende. die übrigen noch nicht blühend oder bloss in Wurzelblättern; die unmittelbar aneinander gereihten sind nach Koch's Synopsis geordnet. 
und die Natur hat ihn, wie alle Schieferberge, mit einer dichten Decke grüner Pflanzen überzogen. Uns gegenüber aber war er weniger freigebig. und mag diess wohl ebenfalls die Jahreszeit rerschuldet haben. Wir trafen beim Hinaufgehen $†$ Draba verna L., Lepidium ruderale L., *Stellaria Holostea L. und *media Vill., *Potentilla rupestris L., argentea L., *Geranium molle L., *Poa bulbosa L. r. vivipara, *annua L. und ron der Spitze gegen den Dom hinunter noch *Sisymbrium Alliaria Scp., Acer campestre L., *Saxifraga tridactylites auf Mauern, *Adoxa Moschatellina L., *Lamium Orvala L., *Arum maculatum L., endlich †Galanthus nivalis L. und ${ }^{*}$ Scilla bifolia $\mathrm{L}$.

Somit ist unsere Ausbeute aus der Umgebung Laibach's erschöpft, denn die Partie in das pflanzenreiche Ischkathal, welche wir uns auf die Rückreise verspart hatten, musste in Folge des anhaltend schlechten Wetters unterbleiben.

\section{Ueber das Karstplateau.}

Vou Laibach geht der Schienenweg lange Zeit ïber grüne Felder, welche grünbelaubte Hïgel umrändern, hinaus zum Moore und quer durch die weite, ebenfalls grüne oder stellenweise blossgelegte Moorfläche hinüber nach Franzdorf. Von da begann sich der Weg allmälig zu heben und bald umschloss uns ein dichter, stämmiger Tannenwald, in dessen Schatten wir nicht selten die krainerische *Scopolina atropoides Schlt., sehr häufig den Zwerghollunder (Sambucus Ebulus L.), äusserst gemein aber die herrliche *Omphalodes verna $\mathrm{Mnch}$. mit ihren grossen azurblauen Blüthen zu schauen bekamen. Dieses niedliche Blümchen begleitete und entzückte uns noch mehrere Stationen weit durcb den "Waldkarst" bis über Rakek hinaus, und ausser ihm notirte ich noch andere Seltenheiten, nämlich *Hacquetia Epipactis DC., *Thlaspi praecon: Wlf. und um Rakek Euphrasia lutea L. Endlich lag die Passhöhe der "julischen Alpen“ (circa $1900^{\circ}$ ) hinter uns und wir hielten ob Adelsberg, dessen Schlossberg wir zu besuchen gedachten.

Auch seine Flora war noch weit zurück und so erschienen die ohnehin ziemlich kahlen Kreidefelsen noch viel kahler und öder. Wir stiegen unmittelbar vom Markte auf und fanden an dem häufigen Steingemäuer Sedum hispanicum L., sexangulare L., *Saxifraga tridactylites L., Hymenostomum tortile Jur., Barbula muralis $\mathrm{Hdw.,} \mathrm{Grimmia} \mathrm{apocarpa}$ $\mathrm{Hdw}$. und Orthotrichum anomalum $\mathrm{Hdw}$. Der Berg selber bot †Helleborus dumetorum Kit., *Arabis arenosa Scp., *Thlaspi praecox Wlf., *Geranium dissectum L., *Cytisus hirsutus L., *Prunus Mahaleb L., *Potentilla verna L., *hirta L. $\gamma$ pedata Wlld., *Poterium Sanguisorba L. v.? polygamum W. K., *Aronia rotundifolia Prs., Eryngium amethystinum L., Peucedanum Oreoselinum Mnch., *Gentiana aestiva R. et Sch., *Myosotis 
hispida Schlechtend., *Teucrium Chamaedrys I., *Globularia vulgaris L., Daphne alpina L. (ganz unentwickelt), *Aristolorhia pallida W1ld., *Mercurialis ovata St. u. II P. P., "Orchis mascula I., "Carex Michelii IIst., * Poa bulbosa I. und Asplenium Ruta muraria I. Am Wege zur Grotte, die wir spät Abends besuchten, auch noch *Lamium Orvala L.

Am nächsten Morgen fuhren wir durch die weite, ob ihros Getreidebaues - vornehmlich Weizen und Mais - berïhmte Hochebene nach St. Peter. Die steinigen Hïgel, welche die Felder umsäumten und den Schafen treffliche Weide boten, waren geschmückt mit zahllosen Blïthen des weissen Thlaspi priecov IVIf., der gelben Potentilla verna L., der blauen Gentiana aestiva R. et Sch. und der schwarzioletten Anemone montana Ilppe. Zu St. Peter erwarteten wir Herrn v. Tommasini, der mit dem Morgentrain von Triest heraufkam, und nach freudiger Begrüssung ging es nun über den eigentlichen Karst südostwärts gegen Fiume. Die Gegend wurde allmälig immer öder und mehr und mehr stellten sich jene Erscheinungen ein, welche dem Karste seinen traurigen Ruf erwarben und die ich in der allgemeinen Uebersicht sattsam bchandelt habe. Lm uns das Hochplatea noch mehr zu verleiden, überschüttete uns der Himmel auch mit einem gewaltigen Hagelwetter, dessen Geschosse zuietzt Alles ringsum bedeckten. Eine freudige Ueberraschung bot die eocäne Rekkamulde, wo statt der steinigen Hügel saftiggrime Wiesen oder braune Aecker, statt der spärlichen Zerr-Eichen (*Quercus Cerris I.) aber schönlauhige Baumgruppen - meist von Schwarzpappeln. Hainbuchen, Buchen, Grau-Erlen, Manna-Eschen und Eichen - uns entgegentraten; bei Elschane auch prächtige Obstbaumpflauzungen, eine Schöpfung des dortigen Pfarrherru. An Pflanzen nichts besonderes: Hie und da *Orchis Morio L., auf Rainen *Euphorbia Cuparissias L. und andere gaewöhnliche Arten der Sandsteinflora. - Jenseits der Rekkamulde aber begann wieder der Karst und zwar noch viel öder und trauriger als zuror, bis er endlich in seiner vollen Schrecklichkeit auftrat und das ganze Land einem bleichen, fleischlosen Gerippe glich. Aeusserst selten begegnete uns eine kleine Gruppe ron Zerr-Eichen oder Schwarzpappelu, eben so selten auch cine Doline. An minderen Pflanzen notirte ich bloss die immer gleich schöne *Anemone montana II p p. und *Scorzonera austriaca IV. - So mochte es wohl eine gute Weile gedauert haben, da ward es allmälig wieder freundlicher. Häufiges Strauchwerk, in welchem Nachtigallen schlugen, Manna-Eschen, Eichen traten auf und die Dolinen wurden häufiger. Einige davon gläuzten gleich grünen Smaragden, andere waren mit Weinreben bepflanzt, den Ersten seit Sava. In einem Gebüsche, welches wir fliichtig durchsuchten, fand sich eine ziemliche Menge interessanter Pflanzen: Vor Allem die wunderschöne *Paconia peregrina Mill., die muthmassliche Stammpflanze unserer Pfingstrosen, *Thalictrum aquilayifolium L., †Helleborus dumetorum Kit., *Corydalis ochroleuca $\mathrm{K} \mathrm{ch}$., 
*Moehinizia muscosa L., *Geranium sanguineum L., *Potentilla alba L., *Symphytum tulerosum L., *Veronica Chamaedrus L., *Pedicularis acaulis Scp., *Thymus Serpyllum L., Satureja montana L., *Aristolochia pallida Wlld., †Carew tomentosa L. und Moose, ausser oben genannten noch Barbula unguiculata Hdw. und Giommia apocarpa $\mathrm{Hd}$ w.

Hier ist das Ende des Karstplateaus erreicht und da auch der Titel dieses Abschnittes nicht weiter reicht, so muss ich abbrechen und einen neuen beginnen :

\section{Das Littorale um den Golf von Fiume.}

Wie das Herz des Menschen beim Anblicke des Meeres aufjauchzt, welches in seiner rollen südlichen Schönheit um Fiume sich ausbreitet, so scheint auch die Natur darüber aufzujubeln und wird in ihrer hohen Freude selber schön und herrlich. Wohl ist der Boden mit dem bisher betretenen identisch und das Kreidegestein blickt uns auch hier nicht selten schroff entgegen, aber die übrigen Factoren haben sich gänzlich verändert: Südliche Lage, Schutz ror dem Winde, unernüdlicher Fleiss der Anwohner, milde Seeluft - Alles wirkt zusammen, um diesen Küstenstrich zu einem der schönsten auf Gottes Erde, zu einem wahren Paradiese zu machen. Wir haben einen grossen Theil dieses Gebietes durchschant, nämlich die reich cultivirte Strecke ron Castua oben am Rande des Karstplateau's bis hinab nach Fiume, feruer die Wälder zwischen Fiume und Volosca, das herrliche Dragathal ostwärts von Fiume, endlich die Umgebung ron Buccari und Buccarizza bis l’orto Rè. Fast alle diese Partien prangten in gleicher Frïhlingsschöne, nur ostwärts rom Dragathale tritt das kahle Gestein, besonders auf den Höhen, grell hervor und macht sie bloss als Schafweiden benutrbar, doch wird auch dort, freilich mit unsäglicher Mühe, allmälig fruchtbares Erdreich aufgeführt und der kalkliebende Weinstock schlïgt dase!bst schon an rielen Stellen kräftige Wurzeln.

Das Reizendste von Allem waren für uns als Floristen natïrlich die noch unentweihten Wraldhaine, besonders die ron mir und I)r. Kriechbaumer durchwanderten Höhen zwischen Fiume und Volosca. Da fanden wir als Hochbäume rorzüglich die beiden Königinnen dieses Gabietes, die majestätische Flaumeiche (*uercus pubescens WIId.) und die weissstraussige Manna-Esche ("Fraximus Ornus L.), dann einen kaum minder hohen, àukelbiättrigen Ahorn (*Acer monsspessulanum L.), *Ostrya carpinifolia Scp., Carpinus duinensis Scp., *†Populus nigra L., ferner als Unterholz die rothblätrige *Pistacia Terebinthus L., den grünlich bliihenden Perrückenstrauch ("Rhus Cotinus L.), den weissblätterigen Mehlbeerbaum ( Sorbus Aria Crt\%), den rothberigen, oft baumartigen * $J u-$ miperus Oxycedrus L., das Pfaffenkäppchen ( ${ }^{*}$ Exomymus europaeus L.), die 
Steinweichsel ( Prum Matualeb L.), die goldigblumige Krouwicke ( ${ }^{*} C_{0-}$ rmilla Emerus L.), "Rhammus infectoria I. v. rdrialica Asch., Paliurus cruleatus Lam., seltener "Silberweiden und "Weissdorn. Unter ihren Fittichen wuchs eine ebenfalls hïchst interessante, theils farbenprächtige, theils unseheinbare, oft arg mit Stacheln bewehrte Flowa: *Hippocrepis comoac L., * Lathyrus Ciceru I.., "sphaericus Retz, "O).ubus albus L. $\beta$ versienlor Keh., Dictumes Fraxinella L., Chrysanthemum corymbosum L., *Centaurea montrana I. u. v. "axillaris W11d., *Crepis vesicaria L., *Cymenchum contigum K ch. (Rehh. Ahb. MLXXIII. Taf. 27. I.), *Melittis Melissophlullum L. r. albiftora, wharseheinlich identisch mit M. nivea Kerner ron Runkelstein bei Bozen etc., deren Kelche meist etwas kahler sind; "Euphorbia Cyparissia.s I.., Smilax aspera L. mit rorjährigen Früchten, *Trmus communis L., *Ruscus aculeatus L., "Asparayus tenuifolius L. am., Aspholelus liburnicus S c p., "Limodomum abortivum Sw. (ein einziges Exemplar gegen Volosca), auf einigen Waldwiesen auch häufig *Orchis Morio L. und * Ophrys apifera $\mathrm{Hds}$.

Diese Wälder und die darin wachsenden Blumen waren auch von zahlreichen Insekten berölkert, aus denen ich mir besonders die Käfer ausersah, während Herr Kriechbaumer sich mit den übrigen zu schaflen machte. Ich fand (gegen Volosea) an Bäumen und Sträuchen : MeloImtha vulyaris Fabr., Epicometis hirtella L., Diacanthus latus Fabr. (1) Cistela nilidula, Attelabus curculinonoides L., Cleonus ophthalmicus Rossi Diomhyrchus !nerzensis Herbst, geniculatus Germar, mastix Olir., Polydrusus Picus Fabr., sericeus Schaller (diese beiden besonders gemein auf Flaumeichen), Balaninus turbatus $\mathrm{Schönh.,} \mathrm{Bruchus} \mathrm{nigripes} \mathrm{D}$ ahl. (1), Lachnaia lonyipes Fabr., Cryptocephalus flavipes Fabr., nitens L., Luperus tavipes L., rujipes Fabr., Coccinella variabilis Illig., mutabilis Scriba, IIalyzia bis-sex-yuttata Fabr., Chiloconus bipustulatus L., Scymnus crpitatus F a br.; auf Blumen: Anthaxia nitidu Rossi, sepulchralis Fabr., Mordella grisea Frochlich, Clytus mysticus I.., Pachyta collaris L.; längs der Strasse nach Volosca fliegend eine ganz kleine Cicindela campestris L., laufend Dorcadion pedestre L., unter Steinen Ocypus cyaneus Payk., "pratrem sabulosum I., Pedinus helopioides Ge rm., Chrysomela limbata F a br., endlich unter Pferdemist Copris lunaris L., Onthophagus semicomis Pz., Sis!nplus Sichaefieri L., Ammoecins brevis Erichson, Aphodius prodromus $\mathrm{Brahm}$. und vitidulus $\mathrm{Fabr}$.

Auf bloss buschigen oder steinigen Hügeh fand sich ausser manchen der schon genannten *Aethionema saratile R. Br., *Polygala vulgaris L., * Corydalis cehroleucu $\mathrm{Kch}$. (ob Buccari), Drypris spinosa L. (ebenfalls bei B.), *Senecin lanatus Scop. (gegen Castua), Carduus nutans L., Helichrysum anyustifolium DC., *Smyirinum perfoliatum Mill. (im Dragathale); Campanula mramidalis L., Salvia nificinalis. L., *matensis L., Tencrium Polium L., TPrimula officinalis J eq. v. suaveoleis Bert. (ob Buccari), Bd. IXU, Abhandl. 
* Euphorbia fragifera Jan., *Aristolochia Clematitis L., *Rumex pulcher I. und *scutatus L., beide häufig un Buccari, * Ornithogalum umbellatum I, v. tenuifolium Guss. (besonders von Buccarizza nach Porto Rè) und †Stipa pennata $\mathrm{L}$. An Meeresfelsen gegen Buccarizza noch Cakile maritima Scp. und in Strassengräben gegen Volosca, Buccari etc. sehr gemein Nasturtium lippicense 1DC. Die felsigen Partien ob Buccari, sowie der Aufstieg rou Buccarizza nach Porto Rè brachten wir auch mehrere Moose: Hymenostomum tortile Jnr., crispatum Jur., Trichostonum mutalile Schmp., Barbula unguiculata $\mathrm{Hdw}$, tortuosa $\mathrm{IFeb}$. et Mohr, intermedia Brid. (= ruralis $\mathrm{Hdw} . \beta$ rupestris Br. eur.), muralis $\mathrm{Hdw}$, Orthotrichum anomalum Hdw., Anomodon viticulosus Hook. et Tayl., Eurhynchium circinnatum Schmp., an Flechten Thalloidima resiculare $\mathrm{Hffm}$.

Die nächste Umgebung von Fiume ist, wie schon Dr. A. Reuss fi!. in 18. Bande dieser Verhandlungen erwähnte, wegen der hohen, die Weinberge und Gärten umsäumenden Mauerı dem Botaniker nicht alizı günstig, doch bieten selbst diese Mauern mehrere interessante Pflanzen, nämlich das blutrothe, häufig rerwilderte *Antirhinum majus L., die gewaltige Campanula pyramidalis L., *† Oralis corniculata L., *Parietaria diffusa Mk., dje höchst gemeine Grammitis Ceterach Sp., Asplenium Trichomanes L. und mehrere der oben genannten Moose. Oefters waren sie auch von Schlinggewächsen umrankt, ausser der Weinrebe vorzüglich voll Epheuguirlanden oder der Bryonia alba L.? Einen interessanten, allerdings nicht ewig dauernden Fundort bot die Stadt selber in ihren Anschüttungen zur Errichtung des Bahnhofes. Wir trafen da eine ziemliche Menge wohl meist gemeiner und uuschöner Schuttpflanzen: *Ramunculus arvensis L., * + Diplotaxis tenuifolia DC.n *Alyssum calycinum I., *Lepidium Draba L., * tcampestre R. Br., *Reseda lutea L., * Silene inflata S m., Arenaria serpyllifolia L., * + Geranium rotundifolium L., * + molle L., *columbinum L., * †robertianum L., *+Medicago minima Lam. r. graecu Il orn (= mollissima Spr.), *Trigonella corniculata L., *Trifolium procmmliens L., "Lotus corniculatus L. $\gamma$ hirsutus $\mathrm{Keh}$ ( $=$ L. villosus Thuill.), "Hippocrepis comosa I., Eryngium campestre I., "T Scandix Pecten Veneris I., *Galium Aparine L., * Cynoglossun cheirifolium Scp., * Scrophularia canina I. (= chrysanthemifolia II. B.), *laciniata. W. K., *Ajuga reptans I. r. albiflora, Plantago serpentina Lam., * Euphorlia fragifera Jan., *Carex diunlsa Good., tdistans L., Cynodon Dactylon Prs., Avena hirsutir Rth., *Festuca ovina L. a vly., Bromus sterilis L. und Hordeum murinum I.

Am interessantesten für jeden Nordländer, besonders den NichtBotanischen, sind ohue Zweifel ob ihrer zahlreichen Südformen die CulturAnlagen, welche die Menschen allhier geschaffen haben und so mag es erlaubt sein, auch diese in Kürze zu besprechen. Welch' malerischen, nach der ïden, traurigen Karstwïste völlig entzïckenden Anblick gewähren vor Allem die Weinberge in ihrer netten Rebenordnung und ihrem 
saftiggrinen Gelaube! Die Rehen stehen alle schnurgerale, in Reih und Glied zu IIecken gezogen - ein Mittelding zwischen der deutschen Sitte. welche sie an Stöcke schmiedet, und der italienischen, welche sie an hohen Bäumen hinaufzieht. Thre Produkte, besonders die Traube von Costrena, sind sehr gesucht wegen ihres milden Feuers, chenfalls der goldenen Mitte zwischen den matteren deutschen und den gluthreichen Insulaner Weinen. Zwischen den IJecken lachen hochanfgeschossene Weizensaaten oder blïhende Frlosenfelder (Pisum satium L.); den noch ïbrigen Raum erfüllen zahlreiche Fruchtbäume der südlichen Zone, vor Allem der grossblätrige Feigenbaum, der besonders in der Ungebung von Porto Rè prächtige, weit berühmte Früchte trägt, ausserdem der weisse *Maulbeerbaum, dessen häufiges Vorkommen auf lebhaften Betrieb der Seidenzucht uns schliessen lässt; hohe, blähende Kirschbäume, duftendes *Lorbeergesträuch, der traubenhehangene fioldregen ("Cutisus Laburnum L.) -- Alles dieses umgeben ron granen, malerisclsen, oft mit Epheu überrankten, oder auf der Höhe nit dem furchtbaren Paliums bewehrten Manem. Tiefer am Meere begegnet uns die häulig gepflanzt, ehrwürdige Olive, rereinzelt auch die dunkelgrüne Pinie, die trüb aufragende Cypresse und der schwarze Maulbeerbaum. Als Alleebäume endlich um die Stadt bieten uns ihren kühlen Schatten mächtige *Rosskastanien, abendländische *Platanen, *Robinien *Schwarzpappeln, *Nuss-, *Maulbeer- und Zürgelbäume (*Celtis australis L.).

\section{Das Reczina-Thal.}

Oben am Karste, an der Grenze von Kroatien und Istrien, entspringt die Reczina, ein ungestïner Gebirgsfluss, der das gleichnamige Thal seiner Länge nach durchströmt und sich nach einem Laufe von $2 \frac{1}{2}$ Meilen unmittelbar bei Fiume unter dem Namen Fiumera in den Golf ergiesst. Da nur die Felsränder des Thales aus Kreidekalk bestehen, die Thalmulde selber aber zur eocänen Formation gehört und grossentheils von Sandsteinkrume erfüllt wird, so ist seine Flora als hygrophile ron der des $\mathrm{K}$ üstenstriches nicht unbedeutend verschieden und mag es daher besser sein, dieselbe gesondert abzuhandeln. Um dieses Thal zu erreichen, muss man lange Zeit zwischen steilen, gigantischen Felsenwänden auf der herrlichen Luisenstrasse und hoch ob dem schäumenden Flusse fortschreiten, bis endlich ein Weg nach links abzweigt und man ïber eine furchtbar schön situirte Brücke dasselbe betritt. An den Kalkwänden bis hieher fand sich "Arabis Turrita L., "Aethimema saratile R. Br., "Coronills Emerus I., "Aronia rotundifolia, I'rs., "Hicrurium laevinatum Wlld. (eine ob des Standortes seegrüne, schmalblätterige Varietat des mururum L.), und * Sesleria elongata Host, an Wegrändern beson lers gemein Itclichrysum angustifolium D C. und *Serophularia laciniatu W. K. 
Das Thal selber bildet eine reizende Idylle: Ringsumber Felder, Weingärten, stattliche Frucht- oder Waldbäume, grïnumkleidete Hügel und darüber kahlere Felshöhen, in der Mitte aber die fröhliche, glattwellige Reczina, an deren Rande sich mehrere grossartige Mühlen erheben. Hinter der letzten treten die Felswände wieder zusammen und nur durch eine schmale Spalte zwängt sich das Flïschen hindurch, wobei es eine Reihe netter, weisser Kaskaden bildet. Wir stiegen eine ziemliche Weile an den Geländen herum, meist im Schatten hoher Zerreichen, Ahorubäume (*Acer monsspessulanum L. und *ampestre L.), Hainbuchen (*Carpinus Betulus L.), * Manna-Eschen, *Mehlbeerbäume, *Therebinthen, * Haselnussstauden und strauchiger Kronwicken (*Coronilla Emorus L.); die buschigen, üppig bewachsenen Hügel und Waldwiesen boten, besonders gegen die Luisenstrasse hinauf: *Barbaraea vulyaris R. Br., *Peltaria alliacea L., * +Thlaspi perfoliatum L., *Cerastium triviale Lnk. und *silvaticum W. K., *Geranium sanguineum I., *Vicia grandiflora Scp. a Scopoliana Kch., *† Orobus vernus L., *Geum urbanum L., *Anthriscus silvestris $\mathrm{Hffm.,} \mathrm{*} \mathrm{Smyrnium} \mathrm{perfoliatum} \mathrm{Mill.,} \mathrm{Aposeris} \mathrm{foetida} \mathrm{Lss.,} \mathrm{*Pul-}$ monaria officinalis L., *Lithospermum purpureo-coeruleum L., *Galeobdolon tuteum L., Euphorbia dulcis L. a lasiocarpa (nicht die als hier vorkommend angegebene, kahlfrüchtige $\beta$ purpurata), *verrucosa Lam., *Orchis laxiflora Lam., *variegata A 11., *fusea J cq, *Ophrys apifera Hds., *Listera ovata R. Br., *†Museari racemosum Mill., *Festuca ovina L.; auf Felsen und Schutt vor den Mühlen häufig *Corydalis ochroleuca Koch, *Parietaria erecta M. K. und besonders gemein *Lepidium Draba L. Diese Pflanzen gehören fast durchgehends zur Sandsteinzone oder siedeln sich wenigstens auf der durch diesen Stein gebildeten, dichten Humuslage mit Vorliebe an und bilden einen überraschend prächtigen, durch hohen Wuchs und Ueppigkeit bezaubernden Anblick, aber, wie ich oben in der Uebersicht gesagt, wenig eigenthümliche Arten, wenig Raritäten.

An Kätern fand sich im Reczinathale ebenfalls wenig Besonderes: Auf Gesträuchen Epicometis hirtella L., Oxythyrea stictica L., Cantharis rustica Fallen, Omophlus lepturoides Fabr., Lixus Myagri Oliv., Otiorrhynchus goerzensis Herbst, Molytes coronatus L., Phyllobius simuatus Fb.. Phytoecia affinis Pz. (1); auf Blumen Malachius spinipennis Germ., Trogoderma versicolor Creutzer, Crioceris merdigera L. Längs der Luisenstrasse: Aphodius prodromus Brahm., Oxytelus inustus Grav. (sehr häufig) und ein prächtiger Carabus catenatus Dfs. Herr Kriechbaumer hatte das Glück, hier auch die niedliche Ptosima novemmaculata. Fabr. in mehreren Exemplaren zu finden. 


\section{Scoglio di San Marco.}

Dieses Felseneiland liegt sibdistlich von Fiume in der Mitte zwischen Veglia und dem festlande von Kroatien, zu dessen Florengebicte es gerechnet wird; es bildet oin langschenkeliges Dreieck, das mit den Tfern der beiderseitigen Umgehung riemlich parallel läuft und seine Basis gegen Fiume wendet, wïhrend die spitze nach Siidosten zum Canale della Morlacea hinunterschat. Das lnselchen enthehrt noch der immergrianen Pflanzen, wie sie die meisten quarnerischen und dalmatinischen Inseln besitzen, und zeigt einen ziemlich kahlen, stellenweise sogar höchst traurigen Charakter; es erheht sich von Nordwesten gegen Siidosten, fallt gegen Sidwesten steil und felsig ah, gegen Norden aber verflache es ich und verliert daselhst fist ginzlich seinen l'llanzenwuchs. Auf ihm findet sich weder Baum, noch llaus, noch irgend welche Spuren des Anbauc. - es dient bloss als Weideland für die Schafe - aber durch die botanischen Forschungen der Englanderin Madame $x$ mith ist es beribut geworden und so wandten auch wir ihm frohen Herzens unser Schiffchen $z$ u, das wir in Porto Re gemiethet hatten. Schon ist der gleich einem Giurtel es umschlingende Felsenwall erklettert und suchend sticgen wir gegen die Höhe empor, wobei wir zwischen den zahllosen ,Teufel:dornern* (Paliurus reculeatus Jam.) uns mühsam hindurchwinden und eine Menge spitz aufragender Kalktriimmer ïberspringen nussten. Doch die Mühe lohnte sich. Ausser dem Puliurus fand sich an Strauchwerk der soeben blühende Weissdorn (Crataegus monogyna Jeq.), Lonicera etruscu Sari, *Rhus Cotinus L., *Rhammes infectoria. I. V. adriatica Asch. und *upestris Scp., und zwischen ihnen eine zwar nicht iippige, aber hüchst interessante Flora: *Arabis hirsuta Scp., *THesperis laciniata All. (nicht = runinatu W. K.), *ellaria alliacea L., †Thlaspi praces WIf., *Rute divaricata T'en., "Genista ovata IV. K., *Hippocrepis comosu L., *Pisum clatius M. B., Spiraea Filipendula L., Eryngium campestre L., Leontodon saxutilis Rchb., Galasia villose Cass., *Hieracium Pilosella L. $\alpha$ vuluare Kch. u. Rehb., "Convoluulus Cantabricr L., " ${ }^{*}$ Cynoylossum cheirifolium Scp., *Orobanche iruenta Bert., *Salvia pratensis L., *Thymus Serpyllum L. v. angustifolius (=Th. acicularis W. K. nach Neilr.), * † Euphorlia firafera Jan., "Thesium divaricatum Jan., *Arum itulicum Mill., "Ruscus aculeatus I., Smilae tepera I., Asphodelus rumosus L., vor Allem aber in häufigen Exemplaren eine vielumstrittene *Cerinthe, welche Joseh und Tommasin zu alpina Kit. ziehen, während Visian i sie für cine Varietät der minor L. hält.

Auf der Höhe bewunderten wir die weite Aussicht ïber Land und Meer, über Kroatiens Schneegebirge und die Inseln des Quarnero, dann stiegen Herr v. Josch und ich au der steilen, viel zerklüfteten Südwestseite 
linunter, während Herr r. Tommasiui weiter nach Osten ging. Auch dieses Felsgewände bot uns eine ziemliche Menge von Pflanzen, und ob dieselben auch fast durchgehends den öden Klippencharakter der Insel nicht verläugneten, wurden sie uns doch ob ihrer Seltenheit liebe Gefährten. Wir sammelten * Silene inflata Smith r. $\gamma$ olevacea Fic. (Rch b., Abb. כ̈120), *Fumaria agraria I, ag., Althaea cannabina I.., *Vallantia mu:alis L., *Talerianella eriocarpa Dsf. a genuina, * + Sonchus asper Vill. $\beta$ pungens 13 ischoff, Plumbayo eniopuea L., "Allium roseum L. und tiefer unten die ob ihrer meist seegrïnen Blätter dem nahen Meere fast homogenen: *Cakile maritima Scp., *Astragalus Wulfeni Kch. (= dem älteren illuvieus Brnh.), Artemiaia maritima L. und zwar nach Tommasini v. gallica WIId., Centaurea cristata Barth. * Scorronera austiaca WIId. (eine glauke, gedrungene Meerform mit meist breiten, krauswelligen Blättern), *Picridium vulgare $\mathrm{Ds}$ s., *+Taraxacum officinale Wig. $\beta$ glaucescens K ch., Teucrium Polium L. und *Plantago serpentina Lam. Nach lang*em, erfolglosen Suchen unseres Schiffchens und nachdem wir die völige Kahlheit der Nordseite sattsam betrachtet hatten, fanden wir uns endlich wieder zusammen und fuhren zurück nach Fiume.

An Käfer hatte ich auf diesem Scoglio wenig gedacht und daher auch wenig gefunden: Auf Weissdornblüthen sehr häufig Cetonia aurata L., aenea Gyllh. und Epicometis hirtella L.; ausserdem noch Otiorrh. gocizens., Meloë erithrocnemus Pallas (1), mehrere Doicadion pedestre I. und Pedinus helopioides Germ.

\section{Veglia.}

Veglia ist die grösste Insel des Quarnero, da sie etwa 5 Meilen Lïnge und 3 Meilen Breite besitzt. Sie hat zwar wegen ihrer südlicheren Lage eine Menge dem Littorale fehlender Pllanzen, doch reicht sie noch nicht, wenigstens nicht in ihrer nürdlichen Hälfte, in das Gebiet der immergrünen Sträucher; die südliche Hälfte hat schon starke Ankläng'e daran und die südlichsten, von uns leider nicht besuchten Spitzen dürfien wohl schon ganz hinein gehören. Diese südlichen und chenso die östlichen Ränder dor Insel sind, wie man schon ron Weitem bemerkt, grossentheils kahl und ron hohen Bergketten durchzogen, der westliche Theil aber ron Castel muschio bis gegen die Iauptstadt hinuuter gleicht einem fast ununterbrochenen Walde, der dem fremden Besucher übergrosses Entzücken, den Einheimischen aber grosse Vortheile bringt, da sie viel davon exportiren. Doch sollen sie dabei zu wenig rationell verfahren und überhaupt scheint die Insel in der Cultur noch weit zurïckzustehen, da die Häuser meist armselig und verfallen, die Bewohner unrein, die Anlagen aber ziemlich rerwahrlost sind. Letztere finden sich wegen der gegen die Nordostseite anstiirmenden Bora fast nur läng's der Test- und Sïdseite, 
besonders um Porto Malinska und um die Hauptstadt Feglia, und sind. wic ibbrhaut die der Quaruero-Inseln, ron denen des Littoma nicht hedeutend verschieden: Weingarten, deren Reben gewöhnlich in Heckrn oder Gruben, stets aber in geraden linien grezogen werden, dazwischen leguminosen, besonders die Saubohne (Ficia F'abu L.) oder einzeln. Weizensaaten, an Fruchtbäunen besmolers die Feige, der Maulbeerbaum und die wegen der siidlichen Iage sely gemeine Olire. - Alles dieses wieder umfriedet ron zerbröckelnden. olt epheummankten, und auf der IÖ̈he mit dem furchtbaren J'uliurus bewehrten Mauern. Eifriger als der Acker wird das Meer nach Speise durchfurcht; so wird z. B. ron der Maju squinado (Granziola) jährlich um 20.000 fl. gefangen, und da in Veglia während unseres Dortseins auch ein Freitag einfel, so fand die aufmerksame Wirthsfrau Gelegenheit, uns eine ziemliche Menge ron Seethieren rorzusetzen, nämlich einen Seeteufel (Lophius piscatorius), einen Steruseher (Cranoscopus scuber), Salptische (Sparus), Meerischen (Muril ceplialus), schmackhafte Barboni (Mullus larbatus), die genannte Muju squinado und endlich, oder eigentlich zuerst, einell im Reise schwimmendeu Tinteufisch (Calamajo, Sepia loliın). Leider war mein Magen für die Meeresproducte nicht eingerichtet und ich muste mir daher das Vergniigen ihrer näheren Bekanutschaft versagen. Unter den Hausthiereu zeichnen sich die "Zackelschafo" - eine dieseu Iuseln eigenthïmliche Race mit sehr langer, zottiger Wolle und kleinen, ziemlich kahlen Schwänzen - durch ihre Unreinlichkeit aus, berühmt hingegen sind die Veglioten wegen ihrer trefflichen Pierde. Es sind diess kleine, meist braune und etwas struppige Ponys mit dunklerem Schweif und Mähne, sie bilden eine dex tïrkischen verwandte und ebenfalls diesen Inseln eigenthiimliche Rare, die trotz ihrer ausserordentlichen Kleinheit kräftig, lebendig und sehwell auf den Beinen ist. Wir hatten während der Fahrt von P. Malinska nach Veglia himreichende Musse, die Schnelligkeit, aber anch das störrine Wesen dieser netten Dingerchen kemmen zu leruen.

Diese Fahrt ging, wenigsteus in der Höhe des welligen P'iateaus, stets durch grünendes Waldgebiet, und die Bäume oder sträucher, welche dasselbe zusammensetzen, sind nach meinen Aufzeichnumen folgente:

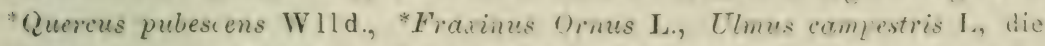
Blätter oft mit rothen Gallenauswiichsen ganz besctzt, †Conus mos 1...

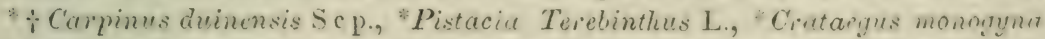
Jcq., selten Pyrus amygdaliformis Vill. Sonst motirte ich noch unter

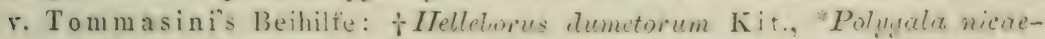

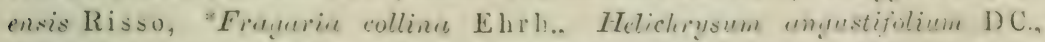

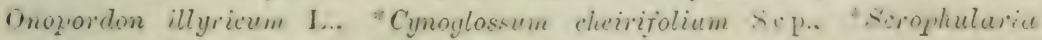
conina L., "Cyclemen repundum Sibt., "Oswivis C.llu L., "Arum italicum Mill.

Von Veglia alis besuchten wir das nordwestlich gelegene Boschetto, ein prächtiges, ebenfalls meist ron "Flaumeichen, "Mauua. Eschen, Feld- 
Ulmen, darunter auch $\beta$ suberosa Ehrh,, dem *Perrïckenstrauche, der *Terebinthe, der Lonicera etrusea Savi, dem Paliurus und Weissdorn gebildetes Wäldchen, aus welchem uns schon von weitem der Kukuk entgegenrief, und in dessen Revier sich eine Menge von blïthenreichen Kleinbiirgern niedergelassen: †Anemone hortensis L. (= stellata Lam.), * Sisymbrium Alliaria Scp., *Polygala nicceensis Risso, *†Cerastium brachypetalum Desp., *Genista ovata Wk., *Anthullis Tulneraria L. r. rubriflora Kch. (= Dilenii), Trifolium angustifolium L., Galega of icinalis I.., *Astragalus glycyplyullos L., *Hipporrepis comosa L., *Vicia angustifolia Rth. $\alpha$ segetalis, *+Eivum hirsutum L., *+Lathyme Aphaca L., *+Cicera L., *pratensis L., latifolius L., *Oenanthe pimpinelloides L., Cnidium apioides Spr., *Chaerophyllum temulum L., Hedera Helix L., *Galium Cruciata Scp., Campanula Rapunculus L., *Lithospermum purpureo-coeruleum I. (sehr häufig), Mentha silvestris L., *Lamium Orvala L., *Melampvruin larbatum W. K., * + Cyclamen repandum Sibt. (äusserst gemein), *Orchis Morio (eine viel stärkere und höhere Form, als die g*ewöhnliche, nach Tommasini $=$ v. Vaiseleri), *Ophrys arachnites Rich. (Waldwiese), † Smilax aspera L., †Ruscus aculeatus L., *Tamus communis L., + Luzula Forsteri DC., † Caree divisa Hds., † Anthoxanthrm odoratum. L., Barbu?a tortuosa W1b. et M., Eurhynchium civinnatum Schmp.

Noch interessanter aber, als das Boschetto, waren die steinigen und buschigen Wegränder, die Raine, besonders aber die verwahrlosten orler aufgelassenen Weingärten, welche sich zwischen dem Boschetto und der Stadt ausdehnen und die wir nach verschiedenen Richiungen durchgangen hatten; viel weniger ergiebig boten sich die knapp ummauerten Wegränder ron der Stadt gegen Cassione hin. Diese Flora war als Schutt- und Rainflora zwar ziemlich unscheinbar, dafür aber desto reicher an Arten und Seltemheiten. Wir fanden: Clematis Vitalba L., *Ranunculus Tommasinii Rchb., *Ficaria L., *muricatus L., *arvensis L., *Fumaria officinalis L., *Papaver Argemone L. (selten), *Cardamine hirsuta. L., *Sisymbrium officinale Sc1., *Alyssum montanum L., tcampestre I., * +calycinum L., *Helianthemum vulyare Grtn. v. concolor Rch b. $(=$ v. hir:sulum Kch.), *Arenaria serpyllifolia L., *†Cerastium pumilum Bön. $\gamma$ riscidum (R chb. Abb. CCXXVIII 4, 969), *Nalva silvestris L., *Erodium “icutarium L'Her., Vitis silvestris Gmel, *Medicago prostrata Jeq., * †orbicularis All., *+Gerardi W. K., * †minima Lam. $\beta$ mollissima Kch., Trifolium *tellatum L., *nigrescens V is., *procumbens L., *Lotus corniculatus L. v. hirsutus Kch., *Astragalus hamosus I., *Coronilla Emerus L., * †cretica L., *Securiger'a Coronilla DC., *Vicia villosa Rth. $\beta$ ylabrescens Kch. (= polyphylla Auct.), *hybrida L., angustifolia Rth. $\alpha$ seg. Kch., varia Host (v. Josch)? *Pisum elatius M. B., *'Lathyrus Aphaca I., * + setifolius L., *Geum urbanum L., Rubus caesius L., *Frayaria collina. Ehrh., Rosa sempervirens I. und canina L.: Ich sammelte Bruchstiicke 
von mehreren Sträuchen - bekam aber leider keine voll-tändigen Exemplare - und unterschied folgende Formen: " vulgaris Keh. = a glabrescens N1r. Die grasgrünen Blattchen, die Bliithenstiele und Kelchröhren gänzlich kahl, die Blattstiele aber überall, nicht bloss an der Basis flaumharig; scheint also ein Tebergang in die var. $\beta$ pubescens N1r. $=\beta$ ilumetorum $\mathrm{K}$ eh. zu sein. Ferner $\gamma$ selosu Meyer (nach Nlr.) $=$ $\gamma$ collina Kch. (collina Jeq. mit Ausnahme der kahlen Blätter). .Blatt-, Blüthenstiele und vorjährige Scheinfrüchte ziemlich zerstrent drüsig borstig, die jungen Blättchen röthlich überlaufen, ältere grün, unterseits bläulich, alle kahl, drüsenlos, einfach gesägt." Endlich $d$ "sepium Kch. (nach Nlr. = muliginoso-canina Meyer). „Blüthenstiele und Kelehröhre bereift, kahl, Blattstiele, Blattränder und unterseitige IIaptadern dicht mit kurzen D!ïsenhaaren besetzt; Blättchen sonst völlig kahl, klein, beiderseits seegrün, unten stärker, doppelt gesägt." Scheint die echte R. sepium Thuill. = myrtifolia IIall. zu sein. - Poterium Santuisorbu L. wahrscheinlich v. polygamum W. K., *Cratue!sus monoyyna Jeq., *Ecballium Elaterium Rich., Bivonia dioica Ja eq. (r. Joseh), "Iferniaria incana Lam. und seltener glabra L., *Sedum acre L., *Sarifraga trirlactylites L., Eryngium campestre L., *广Tordylium apulum L, *Torilis nodosa Grtn., *Cornus sanguinea L. (nach Josch), "Sherardia arvensis L., *Galium Aparine L., "Mollugo L., Scabiosa columbaria L., Artemisia Absinthium L., "Anthemis arvensis L., Cardurs pyonocephalus Jeq., nutans L., Centaurea Calcitrapa L. (sehr häufig auf Rainen um den Lago di Campi), cristata Brtl., *† Rhagadiolus stellatus Grtn. $\beta$ edhulis W., "Liospermun Dalechampii Dsf., *Galasia villosa Cass., Chondrilla juncea L., "† Trichocrepis bifila $\mathrm{V}$ is. (eine nach $\mathrm{R} \mathrm{chb}$. ron Pterotheca nemausensis $\mathrm{Cass}$. gut verschiedene, nach Andern mit derselben identische Gattung und Art), * + Crepis cermua Ten., *Hieracium praealtum $\mathrm{Kch}$. (r. Josch), *Specularia lyyluida DC., Ligustrum vulgare L., *Convulvulus Cantabrica L. selteu, * + Asperugo procumbens L. (neben den Stadtmauern), ${ }^{*}+$ Cymoglossum cheirifolium Scp., Echium pustulatum Sibth., Lithospermum officinale L., Physalis Alkekengi L., *Hyoscyamus albus L. (neben den Stadtmauern), *Verbascum phocniceum L. (gegen Cassione häufig), *† Veronica arvensis L., Digitalis laevigata IV. K., *Orobanche Galii Duby (nicht selten aut Gal. Molluyo), *Tnana Noë (Plitipaea n. Rchb. fil.), auf Sicubinsa columbaria, nach Visiani bloss Varietät der ramosa L., sehr selten, "Salvia prutensis L., *Glechoma hirsuta W. K., Marmbium vul!are L., "Teurvinm chamaed.ys L., *' Cyclamen repandum Sibt., Plumbays europaea L., "Rumex pulcher L., *Osyris alba L. sehr häufig, *Aristolochia Clematitis L., "† Euphorbic Wulfenii H p p. (gegen Cassione), † Mercurialis perennis L., *Celtis australis L. (einzeln), *Arum italirum Mill., *Asparagus tenuijolius L a m., acutifolius L. mit vorjährigen Früchten, *Tamus communis L., *Ginithogalum pyirenaicum L., "Allium roseum L., † Carex divulsa Good., "divisa Bd, XX11. Abhand. 
Hds., †muricata L. (Josch?), Piptatherum multiflorum Bv., Avena hirsuta Rth., Poa dura Scp., *pratensis L., *bulbosa L., coniinna Gd. (vou Josch schriftlich angegebeu mit ?), *Festuca rigida Kunth., Bromus erectus $\mathrm{Hds}$, intermedius Guss. (= confertus M. B.), Lolium italicum A. Br. (Josch), Asplenium Adiantum nigrum L. auf Weingartenmauern.

Im Boschetto, sowie längs dieser Wege und Raine machte ich auch eine bedeutende Ausbeute an Käfern, besonders unter Steinen, nämlich: Anchomenus prasinus F abr., Harpalus ruficornis F a br., semiviolaceus Dej., serripes Schoenh. (sehr häufig), sulphuripes Germ. (sehr gemein), Olisthopus glabricollis Germ. (1), Quedius impressus Pz., Pedinus helopioides Germ. (sehr häufig), Opatrum sabulosum L., Otiorrlynchus giraffa Germ. (häufig), perdix Oliv. (1); auf Gesträuchen: Oxuthyrea stictica L. (sehr gemein), Omophlus lepturoides Fabr., Malachius spinipennis Germ., Attelabus curculionoides L., Rhynchites auratus Scop., Polydrusus flavipes D ege er, Otiorhynchus goerzensis Hrbst. (sehr häufig, rom Volke wegen der grauen Farbe "fratè" genanut), mastix Oliv., Lachnaia longipes $\mathrm{Fbr}$. (besonders auf Flaumeichen), Cyaniris cyanea Fabr., Gynandrophthalma aurita L., Cryptocephalus Hïbneri Fabr. (1), bipunctatus L., Luperus rufipes Fabr. und ein ? Miller rom Boschetto, ähnlich dem cyaneus Dej., Coccinella septempunctata L.; auf Blumen: Agrilus convexicollis Redt., Dulichosoma nobile, Mordella aculeata L. und grisea Fröhl., auf Marmub. vulg. sehr gemein Chrysomela menthastri Suff., eine Var. davon mit glatten Rippen auf den Flügeldecken $=\mathrm{v}$. costata mihi, und cribrosa Germ., auf Weingartenmauern mehrmals Timarcha pratensis Meg., unter Pferdemist sehr gemein Oxytelus inustus Grav.

Auf Veglia sammelten wir auch mehrere Pflanzen der Sumpfflora. Am Wege zum Boschetto liegt nämlich der Lago di Campi, eine grosse, nit quackenden Fröschen erfüllte und fast vollständig mit Schilfrohr bewachsene Feldlache, in welcher sehr häufig * Ranunculus aquatilis L. $\varepsilon$ Petiveri Kch., Callitriche truncata Gussoni (eine Pflanze Calabriens und Siciliens, die nach Tommasini's Mittheilung hier ihren nördlichsten Standpunkt hat, nach Bertoloni bloss eine Var. der C. autumnalis L.), *Potamogeton natans und *crispus L. schwamm; an den Rändern standen rorzüglich *Pulegium vulgare Mill. und *Plantago altissima L. Herr v. Tommasini besuchte auch die Doline Panighe, eine weite, von braunen Aeckern, dann weiter drinnen von grünsumpfigen Wiesen umgebene, und ron einen stagnirenden Wasser erfüllte, pfannenartige Mulde, die wir auf unserer Fahrt ron Malinska nach Veglia gesehen hatten, und brachte uns von dorther den seltenen *Ranunculus ophioglossifolius Vill.; Isnardia palustris L. fand er leider noch zu wenig entwickelt. Dafür brachte er noch aus der Ungebung derselben * Orchis provincialis Balb., *variegata All., * +Ajuga Chia Schr. und *Aristolochia rotunda L. Ebenso verdanke ich seimer und Herrn v. Josch's Güte die auf Meeresfelsen bei 
Veglia gesammelte, wohlriechende "† Ruta bracteosa DC. und die violetblüthige Cakile maritima Scp.

Endlich am Nachmittage vor unserem Scheiden besuchten wir noch Cassione. Es ist diess ein Franziskanerkloster. welches etwa eine Stunde östlich von Veglia auf einer kleinen, rings ron dem tief in's Land eindringenden Meere umschlossenen Insel liegt. An den Rändern des Busens lachen grünende Weingärten, die Insel aber ist grösstentheils bewaldet. Der weitläufge Klosterpark hat sich nämlich durch die Nachsicht seiner Besitzer allmälig in einen förmlichen Wald umgewandelt, in welchem Hochbäume, Cnterholz, Gras- und Kräuterwerk wild durcheinander wächst, so dass man ötters kaum hindurch kann. Der Charakter dieses Gehölzes ist aber schon mehr ein immergrüner, denn das Haupt-Contingent liefern feste Steineichen ( ${ }^{*}$. Ilex L.) und hohes *Lorbeergesträuch; ausser diesen der gemeine *spindelbaum, $+W$ achholder-, $\uparrow$ Mandel-und *IIaulbeerbätume; im Schatten derselben trafen wir besonders *Anthyllis Vulneraria L. var. rubriflora Kch., "Chaernplyllum temulum L., Pallenis spinosa Cass., *Verbascum phoeniceum L., einige Exemplare von *Cynoylossum pictum A it. und häufiger *Asphodelus albus Mill. Die kleinen Erbsen-, Linsen-, Saubohnen-, Getreide- und Weinptlanzungen waren ebenfalls, entsprechend der Sitte des ganzen Eilandes, ziemlich verwahrlost und trafen wir in letzteren Biomus sterilis L. wie angebaut. Auf den Mauern wuchs der seltene, erst im südl. Italien häufig werdende *Umbilicus horizontalis DC. in ziemlicher Menge. Am interessantesten aber für uns war der allerdings sehr triste, stellenweise von der dïrren Cladonia endiviaefolia Dicks. und furcata Schreb. ganz überwucherte Meeresstrand, da auf dem Iuselchen früher Salinen bestanden hatten; wir fanden daselbst Glaucium luteum Scp., drtemisia caerulescens L., Halimus portulacoides W1lr., Salicomia fruticosa L., Statice cancellata Brnhd. und mehrere mit Blïthen röllig übersäete, wohl nur verwilderte Tamarisken (Tamarix africana Poir).

\section{Cherso.}

Cherso, südwestlich ron Veglia, ist weitaus die längste der quarnerischen Inseln, nämlich um \& Meilen länger als selbst Veglia, steht ihr aber an Flächeninhalt bedeutend nach, da ihre Breite durchwegs ziemlich gering ist und in der Mitte durch das tief hineinreichende Vallone di Cherso fast zu einem blossen Felsenkamme zusammenschrumpft. Die Insel bietet keinen so freundlichen Anblick, wie Veglia, sondern erscheint, besonders wenn man dem ron Anlagen und Ortschaften fast entblüssten Osten naht, als ein wïstes, kahles Felsgebirge, was sie auch meistens ist. Schaubch nannte sic treffend einen riesigen, auf der alten Wahlstatt des Meeres liegeuden Knochen. Besonders kahl ist die nördliche Spitze, 
wo der Monte Syss (2016 ) emporragt und das Hochplateau zwischen den Stidten Cherso und Ossero, welches die Chersonesen mit Recht „Arabia potraea" nennen; doch sind selbst diese traurigen Strecken mit zahlreichen Zackelschafen, dem hier weitaus nutzbringendsten IIausthiere, bevölkert, welche an dem zwischen den Steinen aufspriessenden Grase ein zwar spiirliches, aber schr kräftiges Futter finden. Siidöstlich vom Stätchen Ossero verflacht sich das Gehirge und prangt gleich Veglia im ïppigen Grïn der Strincichen und anderer laubbiume. $\Lambda$ n bebauten Plätzen ist diese Insel eben wegen ilırer Bodenbeschaflenheit viel ärmer als Veglia und ist eigentlich nur die Cultur um den IIafen der IIauptstadt von Bedeutung. Hier ist sie aber auch interessant genug: So weit das Auge reicht, ist auf den Höhen ringsum Alles grau, aber nicht von Steinen, sondern von Oliven; Tausende und T'ausende von Bäumen schauen auf die Stadt hornieder und schon von Alters her war die Olive Cherso's hochberïhmt; haben ja die Venetianer dieso Bucht sogar ihren Oelkrug geheissen. Ausser den Oelbäumen aber prangen allhier noch zahlreiche, dunkelblätrige Feigenbäume und ïppige Reben, theils selbstständig, theils im Schatten der Bäume gezogen. An Saalfeldern hat zwar auch diese Bucht kemen Veberfluss, aber die Ursache liegt keineswegs in der Arbeitsscheue ihrer Anwohner, sondern, wie ich schon in der Uebersicht bemerkte, im Mangel an geeigneten Erden.

Was nun unsere Reise betrifit, so landeten wir am Porto di Smergo und ïberstiegen den etwa $1000^{\prime}$ hohen Bergrïcken, welcher uns von der siidwestlich gelegenen Iauptstadt Cherso trennte. In Cherso schieden zu unserem grössten Leidwesen Herr v. Tommasini und Ierr Dr. Kriechba amer, da manfschiebbare P’llichten nach Triest sie riefen; IJerr von Josch und ich botanisirten hierauf um den IIafen ron Cherso, durchritten das Hochplateau der Insel und erreichten zuletzt das Städtchen Ossero, wo wir ebenfalls nach Pflanzen suchten. Ich muss gestehen, dass trot\% des wïsten Charakters der Insel im Allgemeinen doch die $\Lambda$ usbeute uns sehr befriedigte und jener von Veglia nur wenig nachgab. Am reichsten voll allen war wohl der Abhang von dem Porto Smergo bis zur Höhe hinaul. Dieser Abhang gehört, wie alle südlicher gelegenen P'unkte, zur immergrimen Region der Myrte und unterscheidet sich von den nordwärts, sowie siidwärts gelegenen, minder geschiitzten Felsabhängen der Ostkïste auf das vortheilhafteste durch seine dichte Belaubung. Die Höhen bilden hier gleichsam eine halbe Rotonde, deren linke Seite von uralten, stämmigen Steineichen, die andere aber meist mit niedrigem Stranchwerk und blühenden Hïgelpllanzen bekleidet war; an dieser Seite führte die Strasse empor. 'Zwei Maulthicre trugen unser Gepäck, welches rechts und links vom Sattel hinunterhing, wir selber aber folgten zu Fusse, suchend und schwitzend. 1)as Strauchwerk bestand rorzïglich aus Phillyrea medice L., Myitus italice Mill., tJuniperes Oryealius L., *Rhamme infectoria L. 


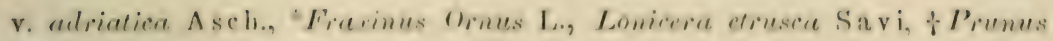
Maludeb I. An nieduren Pllanzen fanden wir *Alyssum momlumm I.,

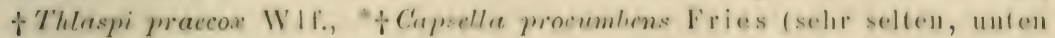

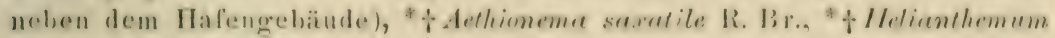
italicum P'rs. (unterscheidet sich von vineale P'rs. durch unterseits griine, fast bloss am Rande und Mitcelnerv striegelhatige Blatter und dureh Slörmig herahgehogene Fruchtstiele - was an den gesammellen fixem-

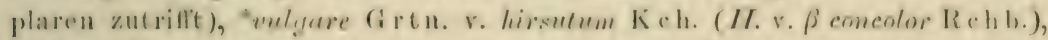

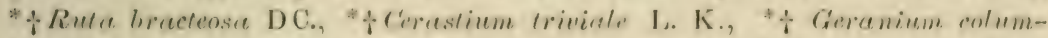

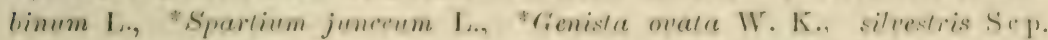

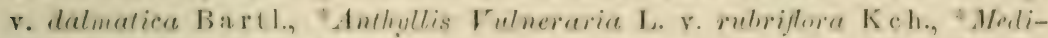

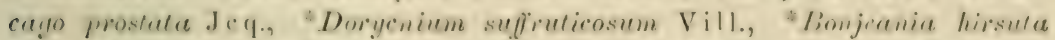

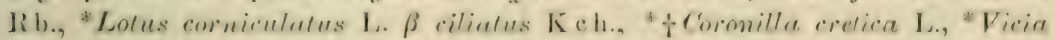

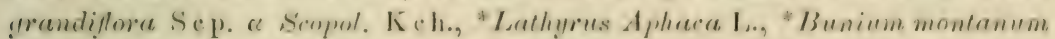
Keh., Cnidium apionides Spr., *Tordylium. apulum. L., Galium lucialum

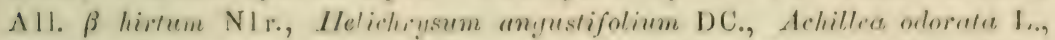

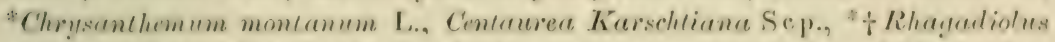

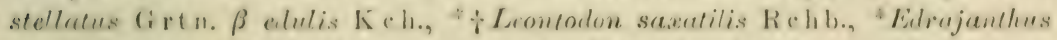
temifolius A. D) C. (aul' den Hähen riemlich selten), "Comoluelus Canfubrica L., "allhreoiles I. ( = temuissimus Sibth.) (boide auch anf der IIöhe ganz neben einander hilulig), * f Cynoglossum cheirifolinm Sep. und "pirtum Ait. (1rsteres besonders diesseits, das zweite jenseits des Bergriickens biiufig), *Onosma stellulatum. W. K., "Lilhospermum purpureo-

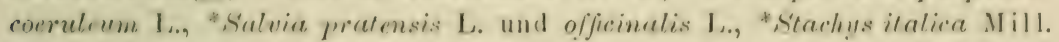

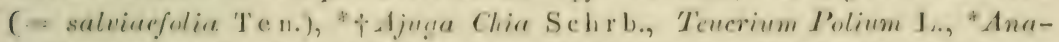
frallis mensis I. (eine kleine, elwas dickblitterige Felsform), "Cyrlamen

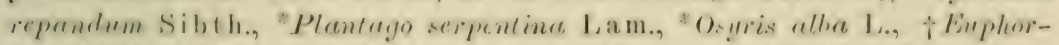
bia Wulfenii Hpp., †xlyrsinies L. (beide gemein), tfragifere Jan., "heliesenpia I.., "Orchis fusea Jeq. (sellen), "Arum ilalienm Mill. und * Carex gynobasis Vill.

Auf der zicmlich kahlen, mit Steinen besäeten llöhe des Bergriickens sammelte ich auch folgende Kafer. Unter Steinen: Calathus

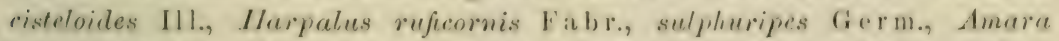
lrivalis Gyllh., Orypus eyaneus l'ayk., Tachimus pallipes Girav., Opu-

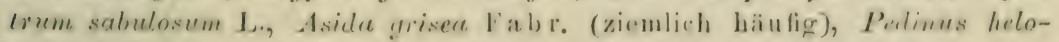
pioides Germ. (sehr häulig), Melops quistuilius Fahr., Mrlö tuerius Rossi, Clemus ophllealmicus Rossi, Lisus Ascanii I. und JunciŚchoenh., Anisorlumelues Mmuchus Germa r (1), Otiorlumchus abuaceus fierm,

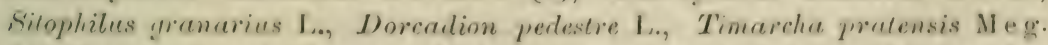
Auf Gestriath und Blumen: Malachius spinipennis Germ., Mimilynchus mastix Oliv., Cimplocephalus sericeus L., Chrysomelu menthastri Suffr., Ciecinella undecimnotala Schn., Scymmus Apetzii MuIsant; endlich unter Dünger: Orntelus inustus tirar., Suprinus conjun!ens Payk., Coprie 
lunaris L., Sisyphus Schaefferi L., Bubas Bison L., Onthophagus Tu.ges Oliv., Schreberi L. (häufig), Lemur Fabr., furcatus Fabr. (häufig). fracticornis Pz., Aphodius erraticus $\mathrm{Fabr}$., Geotrupes laevigatus $\mathrm{Fabr}$., Ateuchus variolosus Fabr., Pentodon punctatus Fabr. An Moosen allhier Hymenostomum tortile Jur. und Barbula intermedia Brid.

Kaum minder reich als dieser Berghang war die Vegetation der Raine und verwahrlosten Weinberge um den Hafen herum. An diesen Lokalitäten fanden wir + Ranunculus muricatus L., * †parviflorus L. (sehr häufig), *repens L., *Delphinum Consolida L. (selten), *Papaver Rhoeas L., * Fumaria parviflora Lam. (in Weingärten ausserordentlich gemein), *+ Cardamine hirsuta L., * + Sisymbrium officinale Sc p., *Lepidium Draba L., * +campestre R. Br., *Tunica Saxifraga Scp., *† Lepigonum medium Whlg. (Kalkgries des Hafendammes), * Erodium malacoides Wlld., †Oxalis corniculata L., *† Medicago lupulina L. $\alpha$ vulg. K ch., * tmaculata W., *Trifolium procumbens L., * + Astragalus hamosus L., * + Coronilla scorpioides Kch. (einige wenige Exemplare in einem Weingarten), * †retica L., * + Securigera Coronilla DC., *Vicia grandiflora Scp. $\alpha$, * +Geum urbanum L., *Potentilla reptans L., * Torilis nodosa Grtı., *+Tordylium apulum L., * Therardia arvensis L., Rubia peregrina L. (auf Weingartennauern hinter dem Hafenende), *Anthemis Pseudo-Cota De Vis. = brachycentros Gay (ein riesiger Stock in einem Weingarten am inneren Hafenende), * Carduus pycnocephalus Jcq., C'entaurea Calcitropa L. und solstitialis L., * + Rhagadiolus stellatus Grtu. $\alpha$ genuinus $\mathrm{Kch}$. (die Achenen gegen die Spitze borstig, sonst kahl), * +Hedypnois cretica Wlld., *Urospermum. picroides Dsf. (kleinere Exemplare, ganzblättrig $=U . \beta$ asperum Duby), *Sonchus asper Vill., * Crepis cernua T en., *tSpecularia hybrida D C., *Symphytum tuberosum L., *Lithospermum purp.-coerul. L., *Hyoscyamus albus L. (an den Mauern des Klosters S. Benedetto), * + Veronica arvensis L., * †serpyllifolia L., * +Orobanche nana Noë auf Shorardia arvensis, *Ajuga genevensis L. flore roseo, *Anayallis arvensis L. und *coerulea Schr., Plumbago europaea L., *Plantago Coronopus L. (auf Kalkkies eines Hafendammes im Hinterwinkel), *Rumex pulcher L., +Euphorbia Wulfenii Hpp., +Peplus L., *Parietaria diffusa Mk. (überall auf Mauern), *Allium roseum L., *+Muscari comosum Mill. (selir gemeiu), † Stipa rennata L., Cynodon Dactylon Prs., *Koeleria phleoides Prs., *Poa bulbosa L. und v. vivipara, *Festuca rigida Kunth, *ovina L. $\varepsilon$ duriuscula Kch., Bromus confertus M. B., rigidus $\mathrm{Rth}$., erectus Hds., Hordeum murinum L., *Aegilops ovata L. Längs des Meeresgestades und während der Fluth von den Wellen bespült fand sich in grosser Menge *Cakile maritima L., Inula crithmoides L., Pulicaria viscosa Cass., Statice Limonium L., und auf der Innenseite, wo sich die Bucht allmälig in einen Sumpf verseicht, sehr gemein *Juncus acutus L., *Scirpus maritimus L. $\beta$ compactus Krock, Taberncuemontani Gmel., †Carex vulpina L., * Textensa Good. und noch 
tiefer drinnen in Süsswasser: *Nasturtium ofjicinale R. Br., Zanichellia palustris L.

Veber das wellige Hochplateau der Arabia petraea war unsere Ausbeute ausserst gering, da wir zu Plerde waren und den Führer bei der Länge des Weges nicht belïstigen wollten; doch wäre sie auch sonst nicht riel bedeutender geworden, eben wegen der enormen Armseligkeit dieses Gebietes. Auf der ganzen, wohl 9 Stunden langen Strecke zwischen Cherso und Ossero fast nichts als wïste Steinflichen oder kahle Felsberge. Dorniges Gestrüpp oder kümmerliches Graswerk senkte zwischen den dürren Kalktrümmern seine Wurzeln hinunter und lechzte nach Wasser; streckenweise fehlte selbst dieses und war die Fläche ganz kahl und öde. Was an höheren Pflanzen oder Bäumen noch hier oben sich angesiedelt, war durch die furchtbare Gewalt der Bora nach Westen gebogen und entbehrte auf der Windseite meist gänzlich des Blätterwerks; so der ziemlich häufige *Juniperus Oxycedrus L., der *Weissdorn, der famose Paliurus, die seltene Pyrus amygdaliformis Vill.; ja selbst die doch so zähen und gewaltigen, hier aber verkrïppelten Steineichen ( $Q$ * Ilex L.) waren über dem Boden oft fast rechtwinklig eingeknickt. An gemeinsten von allen Pflanzen war wohl die Salbei (Salvia ofjicinalis L.), welche besonders zwischen Belley und Ossero die Wïste in zahllosen Exemplaren berölkerte - eine graue Pflanze auf grauem fresteine; ausser ihr begleitete uns streckenweise sehr häufig *Alsine verna Bartl., *Astragalus Wulpenii $\mathrm{K} \mathrm{ch}$. und zwei Wolfsmilcharten ( ${ }^{*}$ Euph. Wulfenii $\mathrm{H}_{\mathrm{p} p .}$. * Myrsinites L.); ziemlich häufig, besonders am Beginn der Wüste, war auch * Cytisus spinescens Sieber, eine diesem Hochplateau und dem Monte Ossero eigenthümliche Pflanze. Was wir sonst noch sahen, war selten und kaum der Rede werth, passte aber rortrefflich in diese Oede: Disteln, Brennesseln, Stechwinden und Brombeergesträuch. So öde und traurig aber anch ringsum Plateau und Gebirge waren, so sahen wir sie doch fast überall mit Steinmauern umgrenzt und in einzelne Weideplätze für die Schafe geschieden - ein Beweis, dass auch hier der Mensch seine Fahue aufgepflanzt und die gottgegebene Herrschaft behauptet hat.

Eine Ausuahme von dieser Schilderung machten nur die wenigen, mit grünen Saaten erfüllten Dolinen und einige grössere Oasen, besonders die Umgebung des schönen Lago di Vrana, welche sogar. Wälder trug, und der Unkreis des Pfarrdorfes Belley. Diese Oasen zeigten dichtgrasige Anger, Wein-, Oliven-, Korn- und Weizenfelder und auf letzterer, wo wir etwas botanisirten, fanden wir die herrliche *Ophrys Bertolonii Mor. sehr bäufig, ausserdem "†Trixayo latifolia $\mathrm{R} b$., ${ }^{*}+$ Linum ancustifolium IIds., "Anthyllis Vulneraria L. v. rubriflora und andere der oben genaunten.

Abends endlich, gegen Ossero hinab, reränderte sich das Angesicht der Erde. Die Anpflanzungen wurdeu häufiger, die graue Salbei trat 
zurïck und eine kleine, grüne Ebene umgab das alterthümliche Städtchen, von dessen Mauern herrliche Epheuteppiche scheinbar herunterhingen. Doch ist auch Ossero so ziemlich eiue Wüste, denn die meisten Häuser sind zerfallen und wir fanden mitten im Städtchen an den Mauern sehr gemein * + Corydalis acaulis Prs., * F Fumaria agraria Lag. Ind †Vaillantia muralis L. a glabra und $\beta$ hispida, welche Varietäten häufig: in eimander übergehen; denn die Stengel sind oft an einer und derselben Pflanze theils obenhin behaart, theils gänzlich kahl. - Auf den öden Schuthïgeln und Ruinen um die Stadt herum fanden wir * + Ranunculus parviflorus L., *Papaver Rhoeas L., Glaucium luteum Scp., *+Vesicaria sinuuta Poir, *Pistacia Lentiscus L., *Trifolium nigrescens Vis., Bryonia dioica J eq., *Ecballium Elaterium Rich., †Tordylium apulum L., Carduus nutans L., *Pieridium vulyare Dsf., *† Crepis rubra L., Phyllyrea media L., * +Lycopsis variegata L., *Echium violaceum L., *Salvia officinalis L., * fclandestina L. (= verbenaca Vhl.), *Stachys italica Mill., Vitew Agnus castus L., Dactylis glomerata L. $\beta$ hispanica Rth. Unterdessen war es dunkel geworden. Der Himmel hatte sich mit Wolken umzogen und der immer stärker heranbrausende Sturmwind trieb uns in die Locanda.

\section{Lossino.}

Lossino (Lussin), die drittgrösste der Quarnero-Inseln, südwestlich ron Cher'so, mit dem seine Nordspitze durch eine etwa $30^{\prime}$ lange Brücke zusammenhängt, bildet ebenfalls ein lang gestrecktes, aber etwas gekrümmtes und noch weit schmäleres Eiland mit zahllosen Buchten und Vorgebirgen; an den beiden Enden verbreitert sie sich keulenförmig und besitzt daselbst die höchste Bodenerhebung, nämlich in Norden den fast zu $2000^{\prime}$ ansteigenden Monte Ossero und im Süden den etwa $800^{\prime}$ hohen Monte Giovauni. Beide Berge sind gegen die Höhe ausserordentlich zerklïtet und ziemlich interesselos, besonders der Monte Ossero, der nach den Berichten der Reisenden fast nichts als endlos wuchernde Salbei besitzt. Um den Fuss dieser Berge aber und längs der Mitte Lussin's ist die Flora wunderbar reich und herrlich, ja weitaus die reichste aller Quarnero-Inseln, trotzdem auch hier die Karstformation durch viele kahle Stellen und scharfe Felsrippen sich genug'sam verräth. Die lohnendsten Fundplätze sind natürlich, wie auf den übrigen Quarnero-Inseln, die buschigen, steinigen Abhänge und die verwahrlosten Oliven- oder Rebengärteu, zu deren Erreichung man die aus Feldsteinen roh aufgebauten Umfassung'smauern übersteigen muss. Wie letzteres vermuthen lässt, ist auch auf Lussin die Laudwirthschaft ziemlich vernachlässigt, aber doch ist diese Insel verhältnissmässig viel reicher cultivirt als das waldreiche Veglia und das gebirgige, steinbesäete Cherso. Das Haupterträgniss liefern Weinstock und Oelbaum. Der Weinstock steht meist in einer Grube 
zwischen kleinen kïnstlichen Hïgeln und sein Laubwerk war während unseres Dortseins schon röllig entwickelt; die Olive aber ist meist etwas rerkïmmert und hatte durch den schneereichen Winter stark gelitten. Ausser ihnen sahen wir besonders Feigen- und *Maubeerbäume ( $\boldsymbol{M}$. alba und niyra L.), *Sorbus domesticu L., Weizensaaten, zwischen denen die Klatschrose (Pupaver Rhoeas L., Aigemone L.) und die Siegwurz (Gladiolus seatum Gaw.) sehr häufig blïhte, und ziemlich viele Leguminosenfelder (*icia Falua L., "Pisum sutizum L., *Cicer arietinum L.); manche der genannten auch rerwildert. Der meisten Pflege erfreuten sich die Abhänge ron Osscro bis gegen Chiunski, vorzïglich aber die um Lussin grande und piccolo, wo auch in den Gärten eine Menge südlicher formen ohme besondere Pllege zu üppigem Gedeihen kommt. Seit Alters beriihmt ist in dieser Hinsicht Lussin grande und die Wirklichkeit blieb hinter unseren Erwartungen nicht zuriick. Wir sahen daselbst prächtige * Citronen- und * † Orangenbäume, *Pinien, immergriune Cypressen, Caroben (Ceratonia siliqua L.), Judasbäume (†Cercis Siliquastrum L.), den P'eifeu-trauch (*Thiladelphus coronarius L.), herrliches *Oleandergebüsch, blïhende Agaven und im Schatten der Genannten eine Menge farbenprïchtiger Blumen. Die daselbst ebenfalls im Freien gezogenen Dattelpalmen und den Paternosterbaum (Melia Azederach) hatten wir leider nicht zu Gesicht bekommen. Bei Lussin piccolo sahen wir an Wegrändern auch den Götterbaum (*Ailantluss ulandulosa Dsf.). Als Curiosum sei noch erwähnt, dass die Brennuesseln auf dieser Insel fehlen solleu.

Was nun die eigentliche Flora betrifft, so hatten wir, um diese kemmen zu lerneu, einen namhaften Theil der Insel durchforscht, nämlich die Strecke ron Ossero bis Lussin piccolo, ron da bis Lussin grande, ferner die Höhen um den Porto S. Martino, den Weg ron Lussin piccolo zum Porto Zigale, endlich den Monte Giovanni, zu dem wir ron Lussin piccolo aufgingen und von dessen Höhe wir nach I. grande himunterstiegen. Unser Führer bei den zwei letztgenannten Partien war Giovanni Gorziu, ein äusserst bescheidener, junger Bursche, den auch Herr von To m masini bei seinen Ausflügen auf dieser Insel bonützt und allen nach ihm Gekommenen empfohlen hatte.

Die herrlichste ron all' diesen Partien war wegen ihres Reichthums an immergrünem Laubwerk und ob des wunderrollen Gesanges zahlreicher Nachtigalleu die ron Ossero nach Chiunski, doppelt herrlich, als wir Tags zuror die "Arabia petraea" durehkostet hatten. Wir salen allhier und zwar meist in Menge: Zwei wunderrolle Cistus-Arten, eine weiss-, eine rothblïhend ( ${ }^{*}$ Cistus salvifolius L. und *reticus L.), †Viburmum Tinus L.. *Quercus Ilew L., *Laurus nobilis L., *Erica arborea L., TJuniperus oxycedrus L. und Trhoenicea 1... Lonicera etrusea Savi, *implexa Ait., TAr-butus T'medo L. (noch grïnbeerig), *Phyllyrea media L., *Pistacia Lentiscus L., Paliurus aculeatus L., Myrtus italica Mill., *Coronilla Emerus L., Bd. IIII, Abhand!. 
vereinzelt auch * Celtis australis L. und an niederen Pflanzen trafen wir läng’s des Weg*es: Clematis Flammula L., *Arabis hirsuta Scp., *Lepidium Draba L., *Reseda lutea L., *+Medicago orbicularis All., *Bonjeania hivsuta Rchb., *Hippocrepis comosa L., *Vicia villosa Rth. $\beta$ glabrescens Kch., †Tordylium apulum L., * + Scandiw Pecten Veneris L., *Herniaria incana I,am., *Helichrysum angustifolium DC., * †Ciepis cernua Ten., *Convolvulus althaeoides L., *Hyoscyamus albus L. (bei Chiunski), *Cynoglossum pictum Ait., *Salvia officinalis I., *Prasium majus L., Teucrium Polium L., *Cyclamen repandum Sibt., *Aristolochia Clematitis L., Plumbago europaea L., tEuphortia Wulfenii Hp p., tfiagifera Jan., *Tamus communis L., *Tris Clusiana Tsch. (in der Gruppe der pallida Lam.), Asparagus acutifolius I., * + Muscari comosum Mill. Läng's des lang*en, schömen Hafencuais ron Lussin piccolo fanden wir ausser vielen der Genanuteu, besouders der hier sehr hänfigen Cistus-Arten, auch noch * Fumaria agraria Lag. und * fofjucinalis L., Glaucium luteum Scp., *Cakile maritima Scp., * Erodium malacoides W1Id., *Sedum acre I., * Orlaya grandiflora $\mathrm{H} \mathrm{ffm.,} \mathrm{*Pallenis} \mathrm{spinosa} \mathrm{Cass.,} \mathrm{Inula} \mathrm{crithmoides} \mathrm{L.,}$ *Urospermum Dalechampii Dsf. (sehr häufig), *†Crepis rubra L., Echium pustulatum Sibth, italicum L.? Viter? Agnus castus L., *Plantago Coronopus I., *Psullium I., *Cynosurus eclizuatus L.

Unser erster Ausflug von Lussin piccolo galt ihrer kleineren aber :̈lteren Schwester Lussin grande. Der Weg führt zuerst durch enge Gässchen und über Stiøgen zur Höhe der amphitheatralisch aufsteigenden Stadt, und ron da auf grandios angelegter Strasse ostwärts dem Meere zu, das wir auch ob dem Valle Darche erreichten. Bis hieher sahen wir auf Gartenmauern häufig verwildert * Matthiola incana R. Br., *Centhranthus ruber DC., * Spinacia inermis Mnch. und auf Schuttboden der Wegraine sehr häufig * +Ranunculus parviflorus L., *Tunica Saxifraga Sc p., * +Geranium rotundifolium L., * +Erodium cicularium L'Her., †Tordylium apulum L., *Helichrys. angustif. D C., *Matricaria Chammomilla L., *Anthemis arvensis L., *Eenecio vulgaris I., *Cardurs pycnocephalus Jcq., *Sambucus nigra L., *Stachys italica Mill., *Euphorbia helioscopia I., * Mrscair comosum Mill., *Arum italicum Mill., *Poa bulbosa L. und r. viripara, Festuca ovma I. und duriuscula Kch., *rigida Kunth, Bromus *confertus M. B., diandrus Curt. (madritensis L.), Hordeum murinum L. Im Valle Darche, einer kleinen, steinigen Thalfäche neben dem weit hereinreichenden Meere trafen wir Glaucium. luteum L., Pulicaria viscosa Cass. (mit verdorrten vorjährigen Stengeln und frischen Trieben), Scolymus hispanicus L., †Vaillantia muralis L., Statice cancellata Brnh., * + Asphodelus ramosus L., *Avena striata Lam., * Briza maxima L. (sehr häufig), *Cynosurnes echinatus I., endlich Dactylis ylomerata L. $\beta$ hispanica Rth. Von da an zieht sich der Weg; äusserst walerisch ob den Fluthen längs der Insel hinuntor. Das Gehänge ist wieder, wie zwischen 
Ossere und Chiunski, ron zahlreichen Lorbeeren, Myrten, den beiden Cistus-, Pistacia- und Lonicera-Arten, hie und da anch einzelnen MannaEschen oder verwilderten Feigen- und Granatapfelbäumen bedeckt und dazwischen entfaltet sich eine artenreiche, dem Nordländer fast gänzlich unbekannte Flora, ron der ich nur das auf Lussin bisher noch nicht notirte eriähne: "f Fumrerie purvifora Lam., "Reserla Plnyteuma L.,

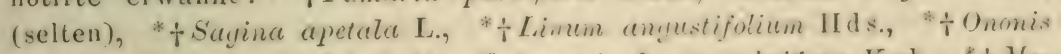
reclinata L. (selten), "Anthyllis V'ulneraria J. r. rulviflom $\mathrm{Keh}$., "t.Me-

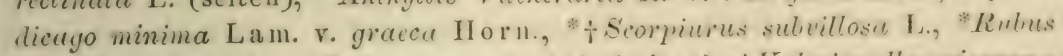
tementosus Borkh., fGulium muale All. (selten), †Valerianella erioearpu 1)sf., Filago germanica L. $\beta$ caneseens Jord., "†Rhaygatiolus stellutus

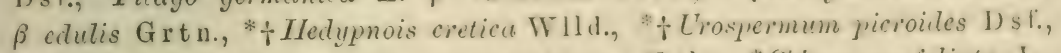
"Hiciacium praeultum Keh. a florentiaum Keh., "Chlora perfoliatu l.., * Erythraea Centuurium P'rs., *onosma stellulatum W. K., Micromeriu juliana Benth., "† Sideritis romana L., "Trasium majus L., "†Ajuga Chia Sehr., *Globularia vulyaris L., *Ophrys apijera Hdw., *Ruscus aculeatus L., Smilax aspera L., † Caree glanca Se p., Birachypodium distuchyon R. et S., Bromus erectus $\mathrm{Hds}$., *Aegilops ovata $\mathrm{L}$. und triuncialis $\mathrm{L}$. (selten). In der Umgebung der Stadt trafen wir Ayave americana L. häufig verwildert und an wïsten Felsrainen gerade vor derselben *Echallion Elaterium Rich. und besonders gemein Glaucium luteum L. Hinter den reichen Gärten L. grande's besuchten wir auch ein verwahrlostes Grundstïck und trafen daselbst + Ranunculus muricatus L., * † partiflorus L., *†Adonis autumnalis L., *Vicia bythinica L. (selten), †Coionilla cietica L., *†Lathyrus Ochrus D C. (selten), *† Zacyntha vermucosa Grti., *Borago officinalis L., *T Serophularia peregrina L., *† Linaria chalepensis Mill., † Euphorbia Peplus L., † Mercurialis annua L., *† Anayallis coerulew Schr. (sehr häufig und üppig).

Der Ausflug auf die Höhen um den ostwärts ron Lussin piccolo gelegenen Porto S. Martino brachte uns ausser vielen schon bekannten, besonders der massenhaft am Meeresstraude wachsenden Pulicariu viscosc

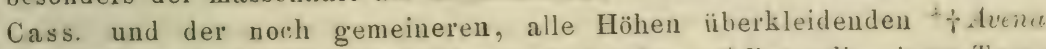
striata Lam. sehr häufig * Tilene sedoides $\mathrm{J}$ eq., *Ruta divericatu Ten., * †rijolium lappaceum L., *† stellatum L., angustifolium L., selten *Ornithoyalum pyrenaicum L. und * Oenanthe pimpinelloides L., äusserst gemein aber gegen die Stadt hiu *Plantago serpentina Lam. Am Wege zum westlichen Porto Zigale trafen wir die schon lang vermissten $t$ Astragalus hamesus L. und + Securigera Coronilla DC., beide in den herrlichsten Sensenfrüchten, ausserdem Kenthrophyllum lanatum DC. und *Tiffolium repens L. v. Biasolettianum; andustifolium L. hier schou in hiibschen, rothen Blütheu.

Die interessanteste aller Partien aber war die auf den Monte Giovanni. Da es die Umstände nicht zuliessen, den M. Ossero zu besteigen 
und auch Herr v. Josch, welcher sich vor 9 Jahren an ihm arg gretäuscht hatte, dazu wenig Lust mehr hatte, so wollten wir wenigstens diese Höhe besuchen, um die Bergflora Lussin's zu besichtigen. Der Weg führte wieder durch die Gässchen der Stadt hinauf und an mehreren KreuzwegStationen, welche zu einem Vorberge dieser Spitze, zum Monte Calvario führeu, vorüber. Endlich blieben alle hemmenden Mauern zurück und wir traten hinaus in's Freie, wo steinige, gelbgrüne Bergweiden und sehr vernachlässigte Olivenpflauzungen uns ein gern gehörtes „Halt" zuriefen. Hier war das Eldorado unserer Wïnsche. Wir trafen: Clematis Flammula L. (an Weingartenmauern), * Fumaria agraria Lag., + Sabulina mucronata L. (Rchb. Abb. 4918 - leider bloss ein einziges Exemplar zwischen Gräsern), Hypericum veronense Schk., *Ononis reclinata L. (sehr selten), * + Medicago orbicularis All., * + Melilotus sulcata Dsf. (sehr häufig), * +Trifolium stellatum L., *angustifolium L., *lappaceum L., *arvense L. $\beta$ strictius Kch., *Lotus odulis L. (einige), *Vicia anyustifolia Rth., *Pisum elatius M. B., * f Scandia australis L. (sehr gemein), *Sedum anopetalum DC. (einige), Filago germanica L. $\beta$ canescens Jord., *Helichrys. angustif. DC., *Urospermum Dalechampii Dsf. (häufig), *+Tragopogon major L. und *porrifolius L., *Chlora perfoliata L., *Erythraea Cent. I., +Veronica arvensis L., Micromeria juliana Benth. (sehr gemein), *Teucrium montanum L. c supinum D e Vis. (1), +Lysimachia Linum stellatum L. (ziemlich häufig), †Euphorbia peploides Gouan. (häufig), *Ophrys aranifera Hds. $\varepsilon$ Tommasinii Rchb., * ${ }^{*}$ Avena striata Lam. (äusserst gemein, die Bergwiesen strohgelb färbend), atherantha = hirsuta $\mathrm{Rth}$, steritis L., * Briza maxima L. (gemein), Poa loliacea Hds. (1), Festuca myurus L. = ciliata Aut. (einige), *Brachypodium pinnatum Bv. $\beta$ rupestre $\mathrm{Kch}$, Bromus mollis L. v. glabrescens, Triticum villosum M. B. (häufig), Asplenium Adiantum nigrum L. (an Mauern). Von da weiter hinauf wurde es aber immer öder und öder; die Mannigfaltigkeit der Formen verschwand und die Salbei überdeckte weite Strecken mit ihrem einförmigen, grauen Mantel; meist aber trat das nackte Felsgestein schroft hervor und zwang: ob seiner mannigfaltigen Risse den Fuss zu kühnen Ansätzen und Sprüngen. Wir sammelten nun einige Fruchtexemplare vou Thlaspi praeco. Wlf. und aus den Ritzen des Gesteins neben einer Mauer holten wir das tief eingewurzelte *Allium subhirsutum L. Fast ebenso arm gab sich die Kuppe: *Trifolium scabrum L., Smilax aspera L. und einige Brombeerstauden. Gegen die Südspitze hinab erstreckten sich Oelpflanzungen und ein dünner Wald von Steineichen. Dafür aber war die Aussicht auf die zahlreichen quarmerischen und dalmatinischen Inseln bis hinunter nach Zara, hinauf nach Fiume, zum Monte Maggiore und Schneeberg, hiniiber zu Kroatiens schneeigen Höhen und endlich westwärts auf das weite, majestätische Meer, wo sich der Himmel mit den Wogen vermählte, überaus entzückend und lohnte die Mühe des letzten Stieges in reichstem Masse. 


\section{S. Pietro di Nembi.}

Pietro di Nembi, eine kleine Insel südlich ron Lussin, ist die südlichste des Quarnero und ihre Südspitze die Südspitze ron ganz Illyrien. Thre nördliche Breite $\left(14^{0} 2 t^{\prime}\right)$ stellt sie so ziemlich in eine Linie mit Bordeaux, Genna und Bologna. Gleich den ührigen Quarnero-Inseln trägt auch Nembi einen ziemlich hohen Berg, den Monte Grisine und zeigt an vielen Stellen den felsigen Karstcharakter. Doch ist es im Ganzen ein prächtig grünes Eiland, reich bepflanzt mit Weinreben und Oliven. Die einzige Ortschaft der Insel ist ein Fischerdorf und wir trafen auch vor Pietro eine ziemliche Menge von Kähnen und Trabaccoli, welche besonder's nach Meerkrebsen (Gronci) und schmackhaften Makrelen (Siombri) fahndeten; das Eingeweide letzterer Thiere muss dazu dienen, um als Köder die übrigen in's Verderben zu locken. Wir landeten vor dem Dorfe, botanisirten etwas in der Umgebung desselben, erstiegen hierauf den M. Grisine und liessen uns von seiner Sputze südlich zum Meere hinunter, von wo wir zwischen Saatfeldern und Oelpflanzungen wieder zum Dorfe gingen. Die Flora musste natürlich im Allgemeinen der von Lussin sehr ähnlich sein und besonders war das immergrïne Laubwerk auf beiden röllig identisch; doch trafen wir eine ziemliche Menge ron Pflanzen, welche wir seither noch nicht gesammelt hatten und auch die schon auf Lussin gefundenen waren in der Menge ihres Vorkommens öfters verschieden. In Nachstehenden gebe ich die ganze Flora, soweit sie uns zu Gesichte kam; leider ist das Verzeichniss nicht so reichhaltig als das des Herrn Dr. Reuss jun. vom Jahre 1867.

Auf Rainen und wïsten Plätzen an der Nordostseite: Eryngium amethystinum I., *Picridium vulgare Dsf., *+Hedypnois cretica W11d., * Lrospermum picroides $\mathrm{Ds}$. und $\beta$ asperum $\mathrm{D}$ uby, "† Asperugo procumbens L. (selten an Häusern), Verbascum sinuatum L., *Stachys italica Mill., † Salix riminalis L.; an Feldnauern Clematis Flammula I., * $†$ Fumaria ayraria Lag., *Rulia pregrina L., Grammitis Ceterach Sw.; in den Feldern selber aber * Fumaria ofjicinalis L., *Althaea hirsuta L. (selten), *Calendula arvensis L. (bäufig), *Asperula arvensis L., *Aristolochice rotunda L. (häufig) und die herrliche, $z$ wischen den Saaten sehr häufige Gladiolus segetum $\mathrm{Gaw}$.

Viel lohnender als die Nord- war die Südseite der Insel längs des Meeres. Wir trafen theils in feinem Dünensande, theils auf den Buschrainen oder zwischen verwahrlosten Reben: $t^{*}$ Fumaria offic. L. v. minor Fries (sehr klein und aufrecht stehend), Glaucium luteum L., "Paparer Rhoeas L., ${ }^{*}+$ Reseda Phyteuma L. (selten), "Malva silvestris L. (häufig), *Medicayo prostrata Jeq." *†orbicularis All., "Tminima L. und v. graeca II orn., *†littoralis Rh., "† Scorpiurus subrillosa L. (am Meere gemein), 
Onopordium illyricum L. (Blätter), * Convoluulus Cantabrica L., *sopium L., *Orobanche minor Sm. auf *Aegilops ovata L., Marrubium candidissimum I., Vitex Agnus castus L., Euphorbia Paralias L. (sehr häufig im Dünensande), *I'lantayo Coronopus L., Psyllium L., serpentina Lam., *Aristolochia Clematitis L. (äusserst gemein zwischen Reben), *+Muscari comosum Mill. (noch gemeiner), *Juncus acutus L., *Phleum arenarium L., ienue L. (1), Arundo Donat L. (verwildert?), *Koeleria phleoides Prs., Dactylis ylomerata L. v. hispanica Rth.; in einem Süsswassersumpfe daselbst, der ganz von Larven wimmelte und mit einer Chara erfüllt war, Zanichellia palustris L. und *Potamogeton crispus L.

Der Berg endlich, welcher zwischen den beiden Inselseiten sich aufbaut, war mit immergrünem Strauchwerk (*Cistus monspeliensis I., *Pistracia Lentiscus L., * + Eirica arborea L., †Juniperus Oxycedrus I., †phoenicea L.) bedeckt, welches besonders auf der Südseite weit ïber Mannshöhe aufgeschossen war und öfters ein fast undurchdringliches Dickicht bildete. Wir fanden an seinen Abhängen * Trifolium stellatum L., ${ }^{*}$ Vicia villosa $\mathrm{Rth.,} \beta$ glabrescens $\mathrm{Kch}$,, ${ }^{*}$ Galium lucidum All. a glabrum (sehr bäufig); +Valerianella eriocarpa Dsf., *Verbascum phoeniceum L., * + Sideritis Romana L., * + Ajuga Chia Schr., * Cynosurus echinatus L. und *Briza maxima L. Aui der Spitze fand sich † Scandia Pecten Veneris L., *Anagallis arvensis L., eine verkiimmerte *Myosotis intermedia Lk.? und eine vereinzelte *Linaria pelisseriana Mill.

An Käfern, die ich seit Cherso arg vernachlässigt hatte - ich notirte bloss rom Lussin grande Orythyrea stictica L. und am M. Giovanni Lina populi L. - traf ich auf diesem Berge Pentodon punctatus Fabr. und Ateuchus variolosus $\mathrm{Fabr}$. unter Kuhmist ziemlich häufig, ferner auf Blïthen Gastrophysa Polygoni L. und sehr gemein Oedemera flavipes F abr., endlich an Steinen Timarcha pratensis Meg.

Auf unserer Rückfahrt stiegen wir in Oriule graude, einer langgestreckten Insel an der Ostseite Lussin's aus und erstieg'en den Rücken derselben, wo uns in einem verwahrlosten Olivengarten * Lupinus hirsutus L. und * Zacyntha verrucosa Gärtn. in Menge entgegentrat, ausserdem als noch nicht gesammelt * Convolvulus arvensis L. und *Plantago lanceolata L. v. hungarica W. K. Ausser dieser Anlage und einer tiefer liegenden, wo wir Marrubium vulgare L. schon in Blüthe trafen, bemerkten wir kein Anzeichen der Cultur, sondern Alles war mit dichtem, immergrünem Strauchwerke überdeckt, worunter sich auch eine Wachholderart mit auffallend grossen, noch grünen Beeren befand - wie uus später Herr v. Tommasin i mittheilte, die echte Juniperus macrocarpa Sibth. Sonst waren wir nicht im Stande, etwas Neues zu entdecken und setzten uns daher wieder in das Schiffchen. 


\section{Zwischen Pola und Promontore.}

Die Umgebung I'ola's bot uns als Reisenden zwei Punkte, welche nach der langen Fahrt ïber steinreiche Inseln ïberaus fesselten und an das schwer vermiste deutsche Vaterland erinnerten, den Kaiserwald und die Pri grande. Ersterer ist ein grosser, von majestätischen Eichen gebildeter, mit Nachtigallen reich berölkerter und von ïppigem, oft lieblich umrankten Unterholz erfüllter Hochwald, die Pra grande aber eine herrlich grünende, mit woissen, gelben und rothen Blïthen übersäcte Wiese, deren früheren sumpligen und fieberschwangeren Charakter man durch Anlegung breiter Kanäle grosstentheils beseitigt hat. Viel weniger aber entziiekten uns diese Lokalitäten als Botaniker, obwohl auch sie manches Neue enthielten. Im Kaiserwalde fanden wir ausser den hohen Eichen (*uercus pseudosuber Saut. nach Tommasini und pubescens Ehrh.), *Acer campestre L., *Cornus sanuminea L., *Lonicera Caprifolium L. (sehr hïufig), Phyllyrea media I. und an niederen Ptlanzen *Vicia grandiflora Scp. a Scopoliana Kch., *angustifolia Rth., *Ervum hirsutum L., *Lathyrus Apliaca L., Orobus niere. L., *Frayaria collina Ehrh., *Sanicula europaea L., Hedera Heli.r L., *Lithospermum purpureo-coeruleum L., *Marrubium vulyare L., *Listera ovata L., "Ruscus aculeatus L., † Care. pallescens I., † silvatica Hds. und sehr häufig *Piptatherum paradonum Br.; auf dem Wege dahin Erhinops Ritro L. und Urtica dioica L. Auf der Pra grande trafen wir sehr häufig *Ranunculus Tommasinii $\mathrm{Rchb.,}{ }^{*} L y c h$ is Hoscuculi L. r. Cyrilli Richter, *onanthe silaifolia Bieb., *Podospermun laciniatum DC., stellenweise auch *Holeocharis uniylumis L.k. und an buschigen Rändern derselben *Physocaulis nodosus Tsch.

Erimnerten diese beiden Stätten lebhaft an die reich bewachsenen Wiesen und Wälder des Nordens, so rersetzten uns die südlich ron der Pra grande durchstreiften, steinigen und starrbuschigen Hïgel wieder ganz in das Gebiet des immergrünen Südens, wie in der That bei Pola die immergrüne Region der Myrte und die sommergrïne der Manna-Esche sich berïhren, da man etwas nördlich ron dieser Stadt das letrte immergriine Buschwerk findet. Die von uns durchwanderten Strecken zwischen Pra grande und dem Monte Gradina lassen sich in das Gebiet der Hïgel und das der Felder eintheilen, wenn auch, wie stets bei solchen Eiutheilungen. sich keine scharfen Grenzen ziehen lassen, da manches beiden gemeinsam ist. Die Felder waren theils mit einer ziemlich dichten Pflanzendecke überzogen und dann boten sie *Dianthus velutinus Guss., *†Lupinus lirsutus I., *Trifolium incarnatum L. mit r. ochroleucum (beide Var. sehr gemein mit zahlreichen Ueburgängen), *† stellatum L., *† Hypochoeris ylabra L., *Galasia villoza Cass., "Picridium vulgare Dsf., *Orchis Morio L. (mit purpurnen und bleichrüthlichen Blüthen), * papilionacea L. (an 
einer Stelle sehr häufig), theils waren sie ärmliche Brachfelder und auf diesen fauden wir * $+R$ anunculus pariflorus L. und * †errucosus Presl. (Rehb. D. Fl. 4618 - wohl bloss eine winzig'e Form des Plilonotis Ehrh.), * + Bunias Erucago L. r. macroptera Rchb.? *Trifolium repens L., *subterraneum L., *angustifolium L., *†Scorpiurus subvillosa L., *† Vicia hybrida I.., *+Alchemilla arvensis S c.p., *†Zacyntha verrucosa Gärtn. (sehr häufig*), * Calamintha Acinos I.., *† Muscari comosum Mill., *Avena capillaris M. Kch.; endlich die Wegränder und die steinigen, mit meist immergrünem Strauchwerk (Phyllyrea melia L., Myrtus italica Mill., * Cistus inonsspeliensis L. (äusserst gemein), Lonicer a etrusca L a v i (Blätter beiderseits behaart), * Rhammus infectoria L. v. adriatica Asch., +Juniperus oxycedrus L., + Acer monsspessulanum L., Clmus campestris L ) besetzten Hiigel enthielten ausser vielen der schon genaunten noch eine Menge vou unscheinbaren, aber meist sehr interessanten Pflanzen, wie ja auch die scheinbar wïsten Buschhügel s der Quarnero-Inseln gerade die reichsten sind. Wir fanden: *Niyella damascena L., *tDiplotaxis tenuifolia DC. (Wegränder), * Senebiera Coronopus Poir. (häufig auf dürrem Lehmboden der Wegränder), Helianthemum Fumana Mill., †salicifolium Prs., *unlyare Grtn. v. concolor Rchb., * Alsine verna Bartl., *Malva silvestris L. V. orbicularis Deth., †Linum angusifolium H $\mathrm{ds}$. v. cribrosum R ch b., *Geranium columbinum L., *Spartirm junceum L., †Medicago orbicularis All., *Trifolium nigrescens $V$ is.. *lappaceum L., *Cherleri L., †Hippocrepis comosa L., *Vicia villosa Rth. r. ylabrescens $\mathrm{K}$ ch., * †angustifolia Rth., * †Lathyrus Cicera L., *Potentilla hirta I. $\alpha$ genuina, †Tordylium apulum L., ${ }^{*} \dagger$ Torilis nodosa Gä rtn., *Galium lucidum All. a glabrum NIr. (= convudaefolium Vill.). *Nolluyo L.? (Josch), Artemisia camphorata Vill. $\beta$ saialilis Kch. und E Biasolettiana Vis., *Cardurts nutans L., Centaurea Calcitrapa L., * +Hedypnois cretica IV 11 d., *Podospermum laciniatum DC. (auf eisenschüssiger, rother Erde flach ausgebreitet, häufig), *† Crepis cernua Ten., *vesicaria L., * Campanula Rapunculus L., *Convulvulus Cantabrica L., Terbascum sinuatum L., *Orobanche minor Sm. $\beta$ adenostyla De Vis., *广 Sileritis romanu L., * Plantayo Lagopus L. (sehr g’emein), *Osyris alba L., *Ophrys aranifera Hds. c atrata Lndl., *Arum italicum Mil., * Ornithogalum umbellatum L., Cynodon Dactylon Prs., Koeleria cristata Prs., Bromus sterilis I. Unter Steinen selten Ocypus cyaneus Payk.

Am Fusse des steinigen Monte Gradina fanden wir neben dem sich weit hereinziehenden Golfe ron Medolino *Plantago Coronopus I. und Euphorbia Paralias L. Der Berg selber bot uns beinahe gar nichts Brauchbares, weil die zahlreich weidenden schwarzen oder weissen Schate nebst kurzhaarigen Rindeın und struppigen Eseln Alles sorgfältig abgefressen hatten, und auch das Plateau von Promontore, welches wir nach Ersteigung dieses Hïgolberges betraten, war äusserst ärmlich. Das ganze Plateau uebst den umliegendeu Höhen war fast uichts als baumloses 
Teideland, nur in Gen diriten ron Promontore fanlen sich Oel-, Feigenund Mande!binme; hinter dem lorfe aber gegen das Meer himab sah man amble griinende Saaten, hesonders Weizen und Mais, mit desen Kolben ancl: die Zimmerdecke unserer Osteria zierlich hehangen war. Tusere ganze Aushente and den mageren Weidegriuden bestand in Trifolium

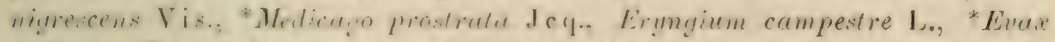

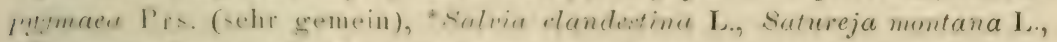

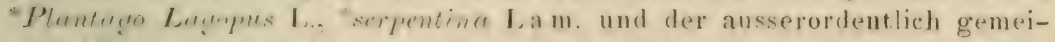

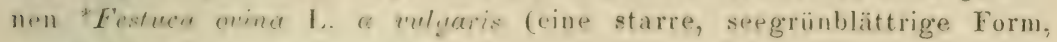
gleich Allen, ron winziger Grösse).

\section{Triest.}

Endlich erreichten wir das letzte Ziel unserer Reise, das freundliche, ruhelose Triest und unseru entflohenen, schmerzlich vermissteu Reiserelahten, Herm r. Tommasini, in dessen fresellschaft wir nun einige shiikliche liage rerlelten. Er war auch so giitig, uns einen Einblick in sein erossartioes Doppelherbar, das allgemeine und das speciell illyri-chu, zu gewahren und die seit seiner Fntferung gesammolten I'llanzen zu hestimmen, wofür ich ihm nochmals unsern wïmsten Dank entrichte. 1) a es ihm nicht mönlich war, uns scine ganze Zeit zu widmen, so machh.n wir unsern ersten Auslug allein, nämlich gegen S. Andrea zu den siidwiirts rou der Stadt am Merresufer gelegenen Anschïttungen, einer fiir Botaniker hochherïhmten Stïtte. Die Kastanien- und Robinien-Alleen. welehe dahin führten, waren ausnehmend hübsch und schattenreich und dwe lliigel neben denselben zeigten zahlreiche wilde oder loch rerwilderte Gesträuche, besnuders spurtium juncerm L.q Colutea arborescens L., Cercis s̈liquastrum L. E'onymus juponica L. fol. rariegat., Li!nustrum unl!are L., Clmus cunpestris L., "Broussonetia papyrifera V ent., als Hecken gerogen "lliliseus suriucus L.; auch cinge Excmplare von * + Smymimu Olusatmu I. dazwischen; die Anschüttungen selber aber boten uichts als lauter gewöhnliche P'llanzen: *Melicagn falcuta I., * + Lepidium Draba I.., Arena falun L. ete. und bestitigten so, was uns Tommasini schon frïhr gasagt hatte, dass sie nämlich jetzt für den Botaniker ganz werthlos seien. Ein starker Regenguss vereitelte unsere Absicht, noch weiter vorzudringen und trieb uus in die Stadt zurïck.

Am nith-ten Tage besuchten wir Miramar, die wunderreiche Schöpfung des Kaisers Max. An den Maueru dahin sahen wir sehr häufig Antirhlinum majus 1. und Contranthes meer DC., beide in den prächtigsten, blutrothen Bhïhen. I) r Farten selher gehört zwar nicht in den Bereich dieser Arbeit, aber als einer der herrlichsten auf Guttes Erde mag er hier ein kleimes l'litzchen finden. Unter allen Gärten ans Oester- 
reich, Deutschland und Belgieu, welche ich gesehen, scheint er mir weitaus der schönste, ja selbst in Italien und Sicilien sah ich keine solche Fïlle exotischer Pflanzen beisammen, als hier. Da wandelt man oft Klafter lang zwischen den herrlichsten Agaveu, da prangen die fremdlïndischen Azaleen, Rhododendren, Weigetien, Kamelien, Magnolien und der strauchige Jasmin in Hunderten der schönsten und vollsten Biüthenkroneu, da steht die chinesische Fächerpalme, da grünen und blïhen die Yucca-Arten, da duftet das weissblumige Pittosporum Tolira in Hunderten ron Sträuchen und bildet schon am Eingange dichte Gehege, da starrt ein ganzer Wald ron spitzen, reich mit Goldblïtheu behangenen Aesten des Spartium junceum I., da überzieht das Hypericum monoynum ganze Abhänge mit ununterbrochenem Blätterdache, da schattet eine Menge ron meist immergrïnen Laub- und Nadelbäumen - Eichen, Cypressen, Thujen, Lorbeer, Myrten, Schling- und Feigenbäume, Araukarien, Wellingtonien, ein ganzer Bestand ron Schwarzführen etc. da ranken die fünfblättrige Ampelopsis, der dunkle Epheu oder die ausnehmend grossblättrige Ficus stipularis an allen Mauern, Felsen und Hallen empor und in den drei Plateaux, welche ïber einander liegen und deren tiefstes das Meer erreicht, da blühen farbenreiche Blumen, da plätschern lustige Foutanen, und dazu schlagen die Sänger iu den Laubgebüschen, dass Einem das Herz fast überfliessen möchte vor Lust und Freude über alı' die Herrlichkeit, welche der gütige Schïpfer den Merschen anvertraut und welche einer der edelsten Söhne unseres Vaterlandes zu einem nnerreichten Wunderbaue zusammengefiigt. Das Lieblichste ron Allem aber war ein Bassin vor dem Schlosse: Auf der Fläche schwammen Wasserrosen mit grossen weissen Blumen und dem goldeueu Stern darinnen, den Rand umduftete blühendes Rosengesträuch, in den durchsichtigen Wellen aber neckten und tummelten sich goldblinkende Fischlein. - Am reichsten unter alleu Pflauzenfamilien sind die Coniferen vertreten, da der Anleger dieses Gartens zu ihnen besondere Vorliebe gefasst und sie daher aus allen Weltgegeuden hieher verpflanzt oder in Samen gezogen hatte und in dicser Beziehung mag der Garten den berühmtesten Gärten der Welt auch an Instructivität wenig nachstehen.

Den dritten Ausflug endlich machten wir auf den Monte Spaccato und diessmal war auch Herr r. Tommasiui dahei. Es ist diess eine 
mässige Karsthühe in Osten ron Triest und der Weg dahin führt anfangs am Fusse des "Boschetto" hin, einer waldigen Berghöhe, welche durch die Sorgfalt der Triestiner von Jahr zu Jahr sich immer üppiger belaubt; dann ging es in Schlangenwindungen allmälig aufwärts zur präclıtigen Terrasse von Rivoltella. Bis hieher sahen wir *Queveus pubescens Ehrh., welche das "Boschettu" grösstentheils bildet, *Rhus Colinus L., "Silene nutuns L. v. livida WIId., *Trifolium montanum I., Oi*obus nigler L., * Lycium barbarum L. (hänfig als Ilecke) und * Onosma stellulatum W. K. Von da an wurden die Irohhen kahler und die Eichen mehr vereinzelt. Die Strasse wandte sich nach links hinüber zu ciner Bucht, in dere.1 Tiefe das Dorf Dongera lag und nach Umfahrung derselben erreichten wir den Monte Spaccato. Von Rivoltella herauf "Polyyala 'u'yaris L., "Hippocrepis comosa L., "Onobrychis Tommasinii Asch., *Galium lucidun All. (sehr gemein), *Rosa rubiginoza L.? *Lenntodon saicatilis $\mathrm{Rehb.}$ tergestinus IIP., "Scrophularia canina I., *Globularia cordifolia I., "Eaphorvia Cyparissias L. (an Wegrändern und auf Veiden äusserst gemein, weil von den Schafen nicht gefressen), ffragifera Jan. Nun folgten wir der Fiumanerstrasse, welche rechts abzweigt, während nıan links hinïber nach Opschina fährt, und bald war die Karsthöhe gewonnen, welche wir als Operationsfeld uns erkoren hatten. Sie war nicht liahl und öde, wie die meisten übrigen Karst-Plateaux, sondern mcist lieblich übergrünt, reich mit Büschen besctzt und stellenweise mit jungen Waldanflügen, nämlich den Schwarzföhren, welche die uncrmüdlichen Triestiner hier angepflanzt. Ensere Ausbeute auf diesen busch- und bliithenreichen Karstwiesen war folgende: † Anemone moniana II P', Rununculus illyricus L., Delphinium fissum W. K., *Pueoniu percitrinu M ill., *Alyssum montanum L., tThlaspi praecor IVIf., *Mochrinetia muscosa L., *Linum Tommasinii Rehb.. *Dictamnus fiacinella I'rs., *Pislacia Terebinllus L., *Genista sericea WIf. (gemein), t.lifïusce W. K., Medicago minima Lam., "ITippoirepis comosa L., *Orobus albus L. $\beta$ versicolor Kch., *Triniu indyaris DC., Ferula Ferulayo L., *Laserquilum siler 1., "Senecio lanatu. Scp. (gemein), Jurinea mollis Rehb., "Leontodon saxat. Rehb., *Trayopoyon Tommasinii Schultz Bip., † Scorroner" austriaca W. a angus:tifolin Mill., Creppis chointrilloides Jacq., "Mieracium sabinum S. et M., "Gentiana aestive R. et Seh., "Cerinthe minor L., *Veronica mullijida L. (= austriacu L. ". bipinnalifula), *Sulvia pratensis 
L., *Plantago Victorialis Poir., *† Convallaria Polyyonatum L., *†Ornithogalum umbellatum L. v. collinum Guss. (nach Koch Syn. II. Ed.!) wohl identisch mit dem O. umb. v. tenuifolium Guss! ron Buccarizza †Fritillaria montana Hp . (= tenella $\mathrm{N}$. B. $), \dagger$ Muscari botruoides Mill Wir hatten im Simne gehabt, in das nahe gelegene Hofgestiite Lippiza zu fabren, allein unterdessen hatte sich der Himmel ganz umzogen und der strömende Regen in Verbindung mit der nordischen Bora zwang uns, sogar das Sammeln aufzugeben; so kehrten wir denn, mit den Erlangten überreich zufrieden, wieder zurück nach Triest und nach lierzlichem Abschiede ron dem aufopfernden Freunde, Ritter v. Tommasini. über die Karsthöhe heim nach Graz und Admont. 


